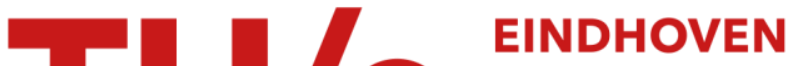 UNIVERSITY OF TECHNOLOGY
}

\section{A monotonicity-preserving higher-order accurate finite-volume method for Kapila's two-fluid flow model}

\section{Citation for published version (APA):}

de Böck, R., Tijsseling, A. S., \& Koren, B. (2019). A monotonicity-preserving higher-order accurate finite-volume method for Kapila's two-fluid flow model. Computers and Fluids, 193, [104272].

https://doi.org/10.1016/j.compfluid.2019.104272

\section{Document license:}

TAVERNE

DOI:

10.1016/j.compfluid.2019.104272

Document status and date:

Published: 30/10/2019

\section{Document Version:}

Publisher's PDF, also known as Version of Record (includes final page, issue and volume numbers)

\section{Please check the document version of this publication:}

- A submitted manuscript is the version of the article upon submission and before peer-review. There can be important differences between the submitted version and the official published version of record. People interested in the research are advised to contact the author for the final version of the publication, or visit the $\mathrm{DOI}$ to the publisher's website.

- The final author version and the galley proof are versions of the publication after peer review.

- The final published version features the final layout of the paper including the volume, issue and page numbers.

Link to publication

\section{General rights}

Copyright and moral rights for the publications made accessible in the public portal are retained by the authors and/or other copyright owners and it is a condition of accessing publications that users recognise and abide by the legal requirements associated with these rights.

- Users may download and print one copy of any publication from the public portal for the purpose of private study or research.

- You may not further distribute the material or use it for any profit-making activity or commercial gain

- You may freely distribute the URL identifying the publication in the public portal.

If the publication is distributed under the terms of Article $25 \mathrm{fa}$ of the Dutch Copyright Act, indicated by the "Taverne" license above, please follow below link for the End User Agreement:

www.tue.nl/taverne

Take down policy

If you believe that this document breaches copyright please contact us at:

openaccess@tue.nl

providing details and we will investigate your claim. 


\title{
A monotonicity-preserving higher-order accurate finite-volume method for Kapila's two-fluid flow model
}

\author{
R. de Böck*, A.S. Tijsseling, B. Koren \\ Department of Mathematics and Computer Science, Eindhoven University of Technology, The Netherlands
}

\section{A R T I C L E I N F O}

\section{Article history:}

Received 20 December 2018

Revised 17 June 2019

Accepted 23 August 2019

Available online 24 August 2019

\section{Keywords:}

Two-fluid model

Hyperbolic system

Finite-volume method

MUSCL

Limiter functions

Source-term treatment

Wave impacts

\begin{abstract}
A B S T R A C T
In preparation of the study of liquefied natural gas (LNG) sloshing in ships and vehicles, we model and numerically analyze compressible two-fluid flow. We consider a five-equation two-fluid flow model, assuming velocity and pressure continuity across two-fluid interfaces, with a separate equation to track the interfaces. The system of partial differential equations is hyperbolic and quasi-conservative. It is discretized in space with a tailor-made third-order accurate finite-volume method, employing an HLLC approximate Riemann solver. The third-order accuracy is obtained through spatial reconstruction with a limiter function, for which a novel formulation is presented. The non-homogeneous term is handled in a way consistent with the HLLC treatment of the convection operator. We study the one-dimensional case of a liquid column impacting onto a gas pocket entrapped at a solid wall. It mimics the impact of a breaking wave in an LNG containment system, where a gas pocket is entrapped at the tank wall below the wave crest. Furthermore, the impact of a shock wave on a gas bubble containing the heavy gas $\mathrm{R} 22$, immersed in air, is simulated in two dimensions and compared with experimental results. The numerical scheme is shown to be higher-order accurate in space and capable of capturing the important characteristics of compressible two-fluid flow.
\end{abstract}

(ㄷ) 2019 Elsevier Ltd. All rights reserved.

\section{Introduction}

Shipment of cargo is a global network of tremendous proportions. The fuels that are currently being used for this purpose are a major source of greenhouse gases. Sustainable energy technology for large-scale ships is still unavailable. In the transition towards clean energy a promising intermediate fuel is liquefied natural gas (LNG), which is obtained by lowering the temperature of natural gas. Its boiling point is approximately at $-160{ }^{\circ} \mathrm{C}$. It is stored around this temperature at ambient pressure in heatisolating cargo containment systems (CCS). It is a mixture of mainly methane and ethane, with small concentrations of heavier alkanes and nitrogen.

The logistical realization of using LNG as a ship fuel requires that huge, seagoing LNG carriers can safely operate at partial filling conditions. Inside CCS complex multiphase mixtures of fluids exist, with possibly violent flow conditions triggered by the ship motion. Sloshing loads during liquid impacts have to be taken into account in the design of state-of-the-art CCS. The most severe impacts occur due to breaking waves slamming onto the wall.

\footnotetext{
* Corresponding author.

E-mail address: r.d.bock@tue.nl (R. de Böck).
}

To study these breaking waves of LNG, the main method is to perform scaled experiments $[4,5]$. Use of these experimental results to predict the full-scale reality requires a scaling law, which is not available as a unified formulation, due to the involvement of complicated multi-scale physics.

First, the tip of the wave reaches the wall, resulting in a short, but powerful acoustic pressure peak. Due to free-surface instabilities, the shape of the wave near the tip is stochastic and unpredictable. This complicates experiments, since no two wave crests created are the same. The wave crest consists of a bubbly flow, so it is compressible. Acoustic waves traveling through the bubbly flow have been observed experimentally [5]. The gas under the crest is compressed, since it cannot escape fast enough from its entrapment between the wave and the wall. This creates a smaller, but longer-lasting pressure on the wall. Next, the gas pressure forces a liquid jet upwards. During this whole process the LNG fluctuates around its boiling point, so phase transition is expected to occur. There is a strong need to better understand the multiphase dynamics involved in liquid impacts.

The ultimate goal of engineers designing LNG-CCS is to understand through numerical simulation, the influence of liquid compressibility, gas compressibility and the density ratio on the pressures exerted on the wall. To model two-fluid flow, a Lagrangian or an Eulerian formulation can be used. A Lagrangian model tracks 
all interfaces explicitly. For complex fluid flow patterns involving many interfaces, the Lagrangian formulation is numerically expensive, since it is an arduous task to track the interfaces. To avoid this difficulty, an Eulerian two-fluid approach will be used in the present work. Various formulations of two-fluid models have been proposed and successfully applied. For several of these formulations it has been shown that they can be extended to include mass and heat transfer across the fluid-fluid interface [7,11,23,24], with application to for instance the study of water-hammer phenomena $[8,9]$. In the current work, we adopt the 5-equation Kapila model [17].

We apply a finite-volume method to the Kapila model, ensuring the global numerical conservation of mass, momentum and energy. At each finite-volume wall we compute the fluxes by means of the HLLC approximate Riemann solver [29]. In the exact two-fluid flow formulation sharp material interfaces exist, however, these are severely diffused when applying a first-order accurate numerical scheme. To mitigate the numerical diffusion, we employ higherorder spatial reconstruction to the finite-volume method, using the MUSCL approach [30]. A limiter function is used to prevent spurious oscillations. We introduce a new limiter function and compare it to commonly used ones.

We consider the one-dimensional test case of a liquid column driven by a body force onto an entrapped gas pocket. This test case is known as the generalized Bagnold model which mimics the impact of a breaking liquid wave onto a solid wall, where a gas pocket is trapped between the liquid and the wall [6].

Furthermore, the impact of a shock wave in air on a bubble containing another gas is simulated in two dimensions. The computational results obtained are compared to experimental results obtained by Haas and Sturtevant [14]. Two cases are considered: one where the gas in the bubble is heavier (R22), and one where it is lighter (helium) than the surrounding air.

The current article is an extension of the paper presented at ECCM-ECFD 2018, Glasgow [10].

\section{Mathematical model}

In this work we study a model that describes a flow consisting of two fluids. Both fluids are assumed to be compressible, inviscid and non-heat-conducting. Furthermore, no chemical reactions or phase changes occur. Analysis of this type of fluid flow has various industrial applications, including sloshing inside LNG tanks [6]. An important issue in the analysis of two-fluid flows is how to account for the presence of each of the fluids and particularly the two-fluid interface. We apply an Eulerian model. To indicate the presence of each of the fluids an indicator function is used. The flow is described by two systems of conservation laws for fluids, two equations of state, and an equation for the indicator function. This type of model is commonly referred to as the Euler-Euler model, or the two-fluid model.

A well-known model of this kind is the Baer-Nunziato sevenequation model [1]. It consists of two systems of conservation laws, one for each of the two fluids. To complete the model, besides two equations of state, an equation describing the presence and proportion of each of the fluids is required: the equation for the volume fraction.

\subsection{The Kapila model}

The Kapila model [17] is a reduced form of the Baer-Nunziato seven-equation model. Kapila et al. argue that for many applications the time scale of equilibration of both velocity and pressure differences across material interfaces is sufficiently small to assume thermodynamic equilibrium, allowing the model to be reduced to five equations. Four of the five equations are conservation

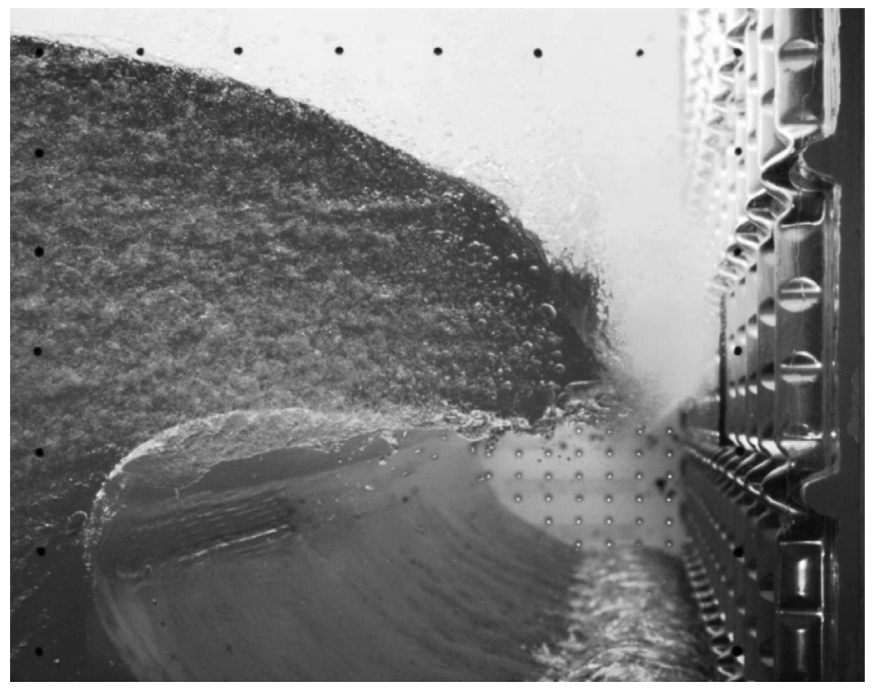

Fig. 1. A breaking wave impacting a CCS-like wall [5].

laws: two for mass, one for momentum and one for energy. The fifth equation concerns the volume fraction of one of the fluids, containing a non-conservative term due to energy exchange between the fluids. Another, equivalent formulation is available [19], with a different representation of the fifth equation.

We will proceed to briefly describe Kapila's two-fluid model. Details can be found in Kapila et al. [17].

The starting point of the model is formed by the Euler equations of mass, momentum and energy conservation for compressible, inviscid, non-heat-conducting fluid flow, without surface tension. This system of equations is given by Toro [29]:

$\left[\begin{array}{c}\rho \\ \rho \mathbf{u} \\ \rho E\end{array}\right]_{t}+\left[\begin{array}{c}\rho \mathbf{u} \\ \nabla \cdot(\rho \mathbf{u} \times \mathbf{u})+\nabla p \\ \nabla \cdot(\rho E \mathbf{u})+\nabla \cdot(p \mathbf{u})\end{array}\right]=\mathbf{0}$.

Here $\rho, \mathbf{u}, E, p$ are the density, velocity vector, specific total energy and pressure, respectively. We also introduce the specific internal energy $e:=E-\frac{1}{2} \mathbf{u} \cdot \mathbf{u}$.

Since we are considering flow of two fluids, this formulation needs to include a way to reflect the varying composition of the combination of fluids throughout the domain. The volume fraction $\alpha_{i}$ is defined as the fraction of a control volume which is occupied by fluid $i$. It takes the value 1 when $(x, t)$ is located within fluid $i$, and 0 when it is within the other. When using averaging methods, these $\alpha_{i}$ can take intermediate values. We assume that the domain is completely filled by the two fluids, resulting in the saturation constraint: $\alpha_{1}+\alpha_{2}=1$. From now on we denote $\alpha:=\alpha_{1}=1-\alpha_{2}$.

An inherent assumption of the model is that any mixing will take place at a scale between molecular scale and macro scale. Fig. 2 shows two examples of two-fluid mixtures. If we suppose these two rectangles are the control volumes used in the definition of the volume fraction, the two-fluid formulation does not see any difference between them, if the volume fraction is the same for both. The control volumes must be sufficiently small for the twofluid formalism to accurately capture the two-fluid flow topology, since any sub-cell flow-topology information will be averaged out over a control volume. The choice of the size of our finite volume cells is therefore especially important.

The quantities used in Eq. (1) are bulk quantities. They are related to the properties of the individual fluids using the volume fraction $\alpha$ as follows:

$\rho=\alpha \rho_{1}+(1-\alpha) \rho_{2}$, 

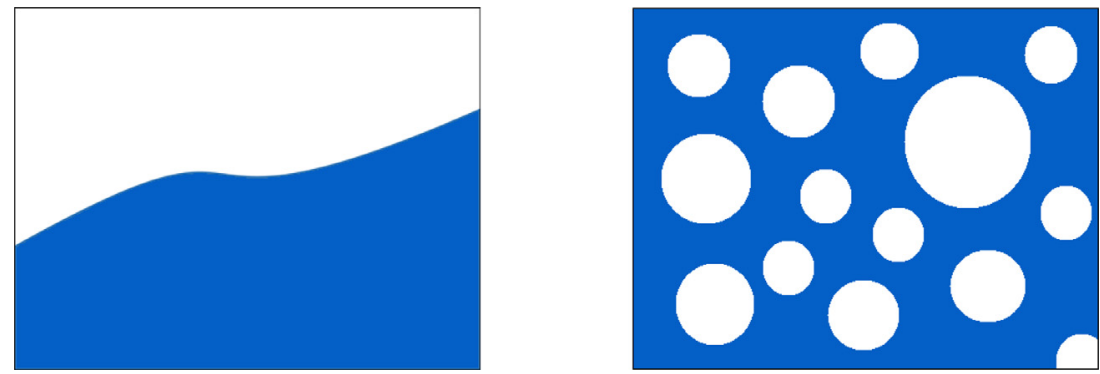

Fig. 2. Two examples of two immiscible fluids in a control volume.

$E=\alpha \rho_{1} E_{1}+(1-\alpha) \rho_{2} E_{2}$

$e=\alpha \rho_{1} e_{1}+(1-\alpha) \rho_{2} e_{2}$.

Because of the pressure and velocity equilibrium assumed across two-fluid interfaces in the five-equation model we have

$p=p_{1}=p_{2}, \quad \mathbf{u}=\mathbf{u}_{1}=\mathbf{u}_{2}$

The Kapila model adds to Eq. (1) the mass conservation law for fluid 1 and an advection-type equation for the volume fraction. The latter contains non-conservative terms which result from imposing the pressure and velocity equilibrium across fluid-fluid interfaces. The system is given by

$\left[\begin{array}{c}\rho \\ \rho \mathbf{u} \\ \rho E \\ \alpha \rho_{1}\end{array}\right]_{t}+\left[\begin{array}{c}\rho \mathbf{u} \\ \nabla \cdot(\rho \mathbf{u} \times \mathbf{u})+\nabla p \\ \nabla \cdot(\rho E \mathbf{u})+\nabla \cdot(p \mathbf{u}) \\ \nabla \cdot\left(\alpha \rho_{1} \mathbf{u}\right)\end{array}\right]=\mathbf{0}$,

$\alpha_{t}+\mathbf{u} \cdot \nabla \alpha+\phi \nabla \cdot \mathbf{u}=0$

Here $\phi$ is a parameter depending on the compressibility of the fluids:

$\phi=\alpha(1-\alpha) \frac{\rho_{1} c_{1}^{2}-\rho_{2} c_{2}^{2}}{(1-\alpha) \rho_{1} c_{1}^{2}+\alpha \rho_{2} c_{2}^{2}}$,

and $c_{i}$ is the speed of sound in fluid $i$. To ease numerical implementation, we write Eq. (4b) in divergence form:

$\alpha_{t}+\nabla \cdot(\alpha \mathbf{u})=(\alpha-\phi) \nabla \cdot \mathbf{u}=0$.

In two dimensions, we have the system:

$\frac{\partial}{\partial t} \mathbf{q}+\frac{\partial}{\partial x} \mathbf{f}+\frac{\partial}{\partial y} \mathbf{g}=\mathbf{s}$,

with $\mathbf{u}=[u, v]^{T}$,

$\mathbf{q}=\left[\begin{array}{c}\rho \\ \rho u \\ \rho v \\ \rho E \\ \alpha \rho_{1} \\ \alpha\end{array}\right], \quad \mathbf{f}=\left[\begin{array}{c}\rho u \\ \rho u^{2}+p \\ \rho u v \\ u(\rho E+p) \\ \alpha \rho_{1} u \\ \alpha u\end{array}\right], \quad \mathbf{g}=\left[\begin{array}{c}\rho v \\ \rho u v \\ \rho v^{2}+p \\ v(\rho E+p) \\ \alpha \rho_{1} v \\ \alpha v\end{array}\right]$,

$\mathbf{s}=\left[\begin{array}{c}0 \\ 0 \\ 0 \\ 0 \\ 0 \\ (\alpha-\phi)\left(u_{x}+v_{y}\right)\end{array}\right]$

This system needs to be closed with an equation of state (EOS) for each of the two fluids, relating the pressure, density and internal energy of each of the fluids. Here we will consider the perfect gas EOS to model gases:

$e_{i}=\frac{p}{\rho_{i}\left(\gamma_{i}-1\right)}$, where $\gamma_{i}$, the ratio of specific heats, is a material constant. The stiffened gas EOS is used to model liquids:

$e_{i}=\frac{p+\pi_{i} \gamma_{i}}{\rho_{i}\left(\gamma_{i}-1\right)}$,

where the liquid behaves as though it is an ideal gas, which is already under a pressure.

\subsection{Analysis of the Kapila model}

In order to analyze the system, we look at the corresponding eigensystem. This gives us information about the wave propagation speeds inherent to the system. In order to do this, we consider an equivalent system, which is expressed in terms of primitive variables $\rho, u, v, p, \alpha, \beta$ :

$\frac{\partial}{\partial t} \mathbf{w}+\mathbf{A} \frac{\partial \mathbf{w}}{\partial x}+\mathbf{B} \frac{\partial \mathbf{w}}{\partial y}=\mathbf{0}$,

$\mathbf{w}=\left[\begin{array}{c}\rho \\ u \\ v \\ p \\ \alpha \\ \beta\end{array}\right], \quad \mathbf{A}=\left[\begin{array}{cccccc}u & \rho & 0 & 0 & 0 & 0 \\ 0 & u & 0 & \frac{1}{\rho} & 0 & 0 \\ 0 & 0 & u & 0 & 0 & 0 \\ 0 & \rho c^{2} & 0 & u & 0 & 0 \\ 0 & \phi & 0 & 0 & u & 0 \\ 0 & 0 & 0 & 0 & 0 & u\end{array}\right]$

$\mathbf{B}=\left[\begin{array}{cccccc}v & 0 & \rho & 0 & 0 & 0 \\ 0 & v & 0 & 0 & 0 & 0 \\ 0 & 0 & v & \frac{1}{\rho} & 0 & 0 \\ 0 & 0 & \rho c^{2} & v & 0 & 0 \\ 0 & 0 & \phi & 0 & v & 0 \\ 0 & 0 & 0 & 0 & 0 & v\end{array}\right]$.

Here, $\beta=\frac{\alpha \rho_{1}}{\rho}$ is the mass fraction of fluid 1 and $c$ is the speed of sound of the mixture, resulting from Wood's relation [32]:

$\frac{1}{\rho c^{2}}=\frac{\alpha}{\rho_{1} c_{1}^{2}}+\frac{1-\alpha}{\rho_{2} c_{2}^{2}}$.

The system, though describing a mixture of two fluids, has a single speed of sound. The speed of sound of a mixture of two fluids can be lower than that of the two individual fluids. The eigenvalues of A and $\mathbf{B}$ are $u-c, u, u+c$ and $v-c, v, v+c$, respectively, where the eigenvalues $u$ and $v$ are both quadruple. The eigenvalues represent wave speeds in $x$ - and $y$-direction, respectively. These are all realvalued, and the eigenvectors of $\mathbf{A}$ are given by:

$\left[\begin{array}{l}1 \\ 0 \\ 0 \\ 0 \\ 0 \\ 0\end{array}\right],\left[\begin{array}{l}0 \\ 0 \\ 1 \\ 0 \\ 0 \\ 0\end{array}\right], \quad\left[\begin{array}{l}0 \\ 0 \\ 0 \\ 0 \\ 1 \\ 0\end{array}\right], \quad\left[\begin{array}{l}0 \\ 0 \\ 0 \\ 0 \\ 0 \\ 1\end{array}\right], \quad \frac{1}{\phi}\left[\begin{array}{c}\rho \\ c \\ 0 \\ \rho c^{2} \\ \phi \\ 0\end{array}\right], \quad \frac{1}{\phi}\left[\begin{array}{c}\rho \\ -c \\ 0 \\ \rho c^{2} \\ \phi \\ 0\end{array}\right]$.

Similarly, matrix B has a full eigenspace, making the system hyperbolic. 
Alternatively, separating the non-conservative part of the equations, we can express the equations as

$\frac{\partial}{\partial t} \mathbf{w}+\tilde{\mathbf{A}} \frac{\partial \mathbf{w}}{\partial x}+\tilde{\mathbf{B}} \frac{\partial \mathbf{w}}{\partial y}+\tilde{\mathbf{C}} \frac{\partial \mathbf{w}}{\partial x}+\tilde{\mathbf{D}} \frac{\partial \mathbf{w}}{\partial y}=\mathbf{0}$,

$\mathbf{w}=\left[\begin{array}{c}\rho \\ u \\ v \\ p \\ \alpha \\ \beta\end{array}\right], \tilde{\mathbf{A}}=\left[\begin{array}{cccccc}u & \rho & 0 & 0 & 0 & 0 \\ 0 & u & 0 & \frac{1}{\rho} & 0 & 0 \\ 0 & 0 & u & 0 & 0 & 0 \\ 0 & \rho c^{2} & 0 & u & 0 & 0 \\ 0 & \alpha & 0 & 0 & u & 0 \\ 0 & 0 & 0 & 0 & 0 & u\end{array}\right]$

$\tilde{\mathbf{B}}=\left[\begin{array}{cccccc}v & 0 & \rho & 0 & 0 & 0 \\ 0 & v & 0 & 0 & 0 & 0 \\ 0 & 0 & v & \frac{1}{\rho} & 0 & 0 \\ 0 & 0 & \rho c^{2} & v & 0 & 0 \\ 0 & 0 & \alpha & 0 & v & 0 \\ 0 & 0 & 0 & 0 & 0 & v\end{array}\right]$

$\tilde{\mathbf{C}}=\left[\begin{array}{cccccc}0 & 0 & 0 & 0 & 0 & 0 \\ 0 & 0 & 0 & 0 & 0 & 0 \\ 0 & 0 & 0 & 0 & 0 & 0 \\ 0 & 0 & 0 & 0 & 0 & 0 \\ 0 & \phi-\alpha & 0 & 0 & 0 & 0 \\ 0 & 0 & 0 & 0 & 0 & 0\end{array}\right]$

$\tilde{\mathbf{D}}=\left[\begin{array}{cccccc}0 & 0 & 0 & 0 & 0 & 0 \\ 0 & 0 & 0 & 0 & 0 & 0 \\ 0 & 0 & 0 & 0 & 0 & 0 \\ 0 & 0 & 0 & 0 & 0 & 0 \\ 0 & 0 & \phi-\alpha & 0 & 0 & 0 \\ 0 & 0 & 0 & 0 & 0 & 0\end{array}\right]$

The eigenvalues of $\tilde{\mathbf{A}}$ and $\tilde{\mathbf{B}}$ are the same as those of $\mathbf{A}$ and $\mathbf{B}$, and the eigenvectors are similar:

$\left[\begin{array}{l}1 \\ 0 \\ 0 \\ 0 \\ 0 \\ 0\end{array}\right], \quad\left[\begin{array}{l}0 \\ 0 \\ 1 \\ 0 \\ 0 \\ 0\end{array}\right], \quad\left[\begin{array}{l}0 \\ 0 \\ 0 \\ 0 \\ 1 \\ 0\end{array}\right], \quad\left[\begin{array}{l}0 \\ 0 \\ 0 \\ 0 \\ 0 \\ 1\end{array}\right], \quad \frac{1}{\alpha}\left[\begin{array}{c}\rho \\ c \\ 0 \\ \rho c^{2} \\ \alpha \\ 0\end{array}\right], \quad \frac{1}{\alpha}\left[\begin{array}{c}\rho \\ -c \\ 0 \\ \rho c^{2} \\ \alpha \\ 0\end{array}\right]$.

Since the solution of the hyperbolic system is generally based on the decomposition of the matrices $\mathbf{A}$ and $\mathbf{B}$, or $\tilde{\mathbf{A}}$ and $\tilde{\mathbf{B}}$, the fact that the eigenvalues of these matrices are the same means that we can freely choose whether to include the non-conservative term in the (approximate) Riemann solver of choice, or to treat it separately. This freedom of choice is exploited by handling the conservative part of the equations with a fully conservative finite-volume scheme and by separately discretizing the non-conservative term.

\section{Numerical approach}

We consider the Kapila system (7a) in integral form, at some point in time, say $t$ :

$\frac{\partial}{\partial t} \int_{\Omega} \mathbf{q} \mathrm{d} \Omega+\oint_{\Gamma} \mathbf{F} \cdot \mathbf{n} \mathrm{d} \Gamma=\int_{\Omega} \mathbf{s} \mathrm{d} \Omega$,

with $\Omega$ a control volume and $\Gamma$ its boundary,

$\mathbf{q}=\left[\begin{array}{c}\rho \\ \rho u \\ \rho v \\ \rho E \\ \rho \beta \\ \alpha\end{array}\right], \quad \mathbf{F}=\left[\begin{array}{ll}\mathbf{f} & \mathbf{g}\end{array}\right]=\left[\begin{array}{cc}\rho u & \rho v \\ \rho u^{2}+p & \rho u v \\ \rho u v & \rho v^{2}+p \\ u(\rho E+p) & v(\rho E+p) \\ \beta \rho u & \beta \rho v \\ \alpha u & \alpha v\end{array}\right]$, $\mathbf{s}=\left[\begin{array}{c}0 \\ 0 \\ 0 \\ 0 \\ 0 \\ (\alpha-\phi)\left(u_{x}+v_{y}\right)\end{array}\right]$

To apply a finite-volume method, we divide the computational domain into rectangular cells, which have an average state $\mathbf{q}_{i, j}$. In semi-discrete form we have

$$
\begin{aligned}
\frac{d}{d t} \mathbf{q}_{i, j}= & -\frac{1}{\Delta x}\left(\mathbf{f}_{i+\frac{1}{2}, j}-\mathbf{f}_{i-\frac{1}{2}, j}\right)-\frac{1}{\Delta y}\left(\mathbf{g}_{i, j+\frac{1}{2}}-\mathbf{g}_{i, j-\frac{1}{2}}\right) \\
& +\frac{1}{\Delta x \Delta y} \int_{\Omega_{i, j}} \mathbf{s}_{i, j} \mathrm{~d} \Omega_{i, j} .
\end{aligned}
$$

The basic update rule for $\mathbf{q}_{i, j}$ is the Godunov upwind method, with explicit Euler time integration:

$$
\begin{aligned}
\mathbf{q}_{i, j}^{n+1}= & \mathbf{q}_{i, j}^{n}+\frac{\Delta t}{\Delta x}\left(\mathbf{f}_{i-\frac{1}{2}, j}^{n}-\mathbf{f}_{i+\frac{1}{2}, j}^{n}\right)+\Delta t \mathbf{s}_{x, i, j}^{n} \\
& +\frac{\Delta t}{\Delta y}\left(\mathbf{g}_{i, j-\frac{1}{2}}^{n}-\mathbf{g}_{i, j+\frac{1}{2}}^{n}\right)+\Delta t \mathbf{s}_{y, i, j}^{n},
\end{aligned}
$$

where the subscripts $x$ and $y$ in $\mathbf{s}_{x}$ and $\mathbf{s}_{y}$ do not refer to partial differentiation of $\mathbf{s}$, but to the following components of $\mathbf{s}$ instead:

$\mathbf{s}_{x}=(\alpha-\phi) u_{x}$

$\mathbf{S}_{y}=(\alpha-\phi) v_{y}$.

We remark that the idea of the directional splitting of the source term is not part of Godunov's original approach; Godunov considered homogeneous equations only. In the numerical evaluation of the split source term, we will make handy and consistent use of the numerical method for evaluating the fluxes (see Sections 3.2 and 3.3).

The finite-volume method is conservative; the discretization does not lead to a global change in mass, momentum and energy. Here, both spatial and time discretization are performed in a higher-order accurate manner, as outlined in the following sections.

\subsection{Spatial discretization}

To achieve higher-order spatial accuracy we perform spatial reconstruction, known as the MUSCL (Monotone Upstream-centred Scheme for Conservation Laws) approach [30]. We present it here for the one-dimensional case. The formulation is easily extended to two- and three-dimensional cases, treating the fluxes in each of the separate directions in a locally one-dimensional fashion.

In the MUSCL approach the states inside the cells are not considered to be piecewise constant. Instead we may reconstruct e.g., piecewise linear distributions of state values inside the cells. It is preferable to reconstruct in this way the primitive variables, rather than the conservative variables, or any other formulation [31]. In each cell we may define a linear function for each of the primitive variables individually:

$W_{i}(x)=W_{i}+\frac{x-x_{i}}{\Delta x} \Delta_{i}, \quad x \in[0, \Delta x]$,

where $\frac{\Delta_{i}}{\Delta x}$ is a suitably chosen slope; the method to choose the slope will be outlined in the following.

This approach allows us to construct higher-order accurate methods. However, a careless implementation would result in overshoots at discontinuities, as sketched in Fig. 4. For the system under consideration this is a very undesirable situation, since we 


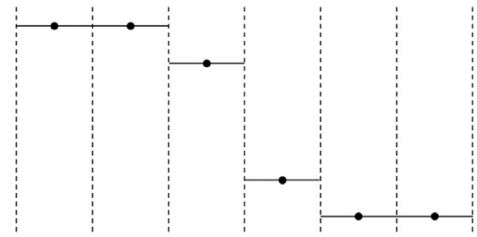

(a) before spatial reconstruction

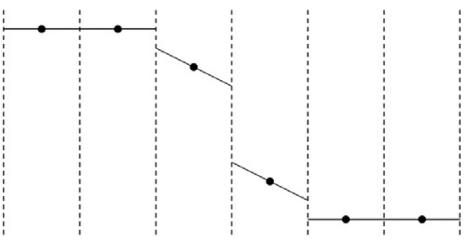

(b) after spatial reconstruction

Fig. 3. The values for a state variable in a number of cells. In (a) the value in the cells is the average, in (b) a linear distribution has been constructed.

have strict demands on our variables: the absolute pressure and density must be positive, because of the calculation of the speed of sound, and the volume fraction must remain in the [0,1]-interval.

We need a numerical scheme that does not exhibit these overshoots and the spurious oscillations that result from it. Monotonicity-preserving schemes form an important class of numerical methods, that do not suffer from spurious oscillations. They have the property that a system with a monotone initial condition will remain monotone in future time steps. This means the scheme mimics a property which is also exhibited by the exact solution of the system [20]. Godunov's theorem states that monotonicity-preserving linear schemes can be at most first-order accurate [13].

To overcome this barrier, we use the more lenient demand of total variation diminishing. To quantify the oscillations we consider the total variation, which is defined for functions with a discrete domain as the sum of the differences between state variables in adjacent cells:

$T V\left(u^{n}\right):=\sum_{i}\left|u_{i+1}^{n}-u_{i}^{n}\right|$

We want this total variation as a function of our variables to be non-increasing for progressing time steps. A numerical scheme that exhibits this property is called total variation diminishing (TVD). TVD schemes are monotonicity preserving, meaning that no new (unphysical) local extrema are created. TVD schemes therefore do not create spurious oscillations.

To prevent the spurious oscillations in the MUSCL method and make the scheme TVD we apply a slope limiter $\varphi$ to the primitive variables $W_{i}$ :

$W_{i+\frac{1}{2}}^{L}=W_{i}-\frac{1}{2} \varphi_{i+\frac{1}{2}}^{L}\left(W_{i}-W_{i-1}\right)$,

$W_{i+\frac{1}{2}}^{R}=W_{i+1}-\frac{1}{2} \varphi_{i+\frac{1}{2}}^{R}\left(W_{i+1}-W_{i}\right)$.

We apply the limiter to each of the primitive variables individually. In the next step the Riemann problem is solved for the limited variables to obtain the fluxes:

$F_{i+\frac{1}{2}}=F_{i+\frac{1}{2}}\left(W_{i+\frac{1}{2}}^{L}, W_{i+\frac{1}{2}}^{R}\right)$,

$F_{i-\frac{1}{2}}=F_{i-\frac{1}{2}}\left(W_{i-\frac{1}{2}}^{L}, W_{i-\frac{1}{2}}^{R}\right)$.

The limiter has the function of switching between a favorite higher-order method of our own choice, for smooth regions of the solution, and the first-order method (corresponding to the piecewise constant solution representation illustrated in Figs. 3a and 4a) in regions with steep gradients. We therefore define it as a function of the adjacent slopes of each of the individual primitive variables:

$\varphi_{i+\frac{1}{2}}^{L}=\varphi\left(r_{i+\frac{1}{2}}^{L}\right), \quad \varphi_{i+\frac{1}{2}}^{R}=\varphi\left(r_{i+\frac{1}{2}}^{R}\right)$, with

$r_{i+\frac{1}{2}}^{L}=\frac{W_{i+1}-W_{i}}{W_{i}-W_{i-1}}, \quad r_{i+\frac{1}{2}}^{R}=\frac{W_{i}-W_{i+1}}{W_{i+1}-W_{i+2}}$.

The theory of spatial reconstruction and slope limiting is usually presented for a 1-D linear advection equation. In that context, we gain insight in the meaning of the limiter:

- Setting the limiter to zero: $\varphi(r)=0$ is the same as simply applying the first-order upwind Godunov method.

- The second-order central method is obtained for $\varphi(r)=r$.

- For $\varphi(r)=1$ we obtain the second-order upwind method.

Spekreijse [26] outlined sufficient conditions for the limiter to be met in order for the method to be TVD. They can be put in the form

$m \leq \varphi(r) \leq M, \quad \forall r \in \mathbb{R}$,

$-M \leq \frac{\varphi(r)}{r} \leq 2+m, \quad \forall r \in \mathbb{R}$.

Here $m$ can be chosen in the $[-2,0]$ interval. The choice of the other parameter, $M$, if chosen to be greater than 1 , causes a time step restriction according to, following [15],

$\mathrm{CFL} \leq \frac{2}{M+2}$

For the method to be fully second-order accurate we require that $\varphi(1)=1[26]$.

Often, the parameters in the TVD condition from Spekreijse are chosen to be $m=0$ and $M=2$. This leads to a region in the $r, \varphi$ plane, called the Sweby region [27]. In this case, for the method not to be overly compressive, the limiter must not exceed the upper bound of Sweby's TVD domain. Excessive compression could for example result in a smooth transition being steepened to a discontinuity.

We give a summary of some commonly used limiters. The minmod limiter is the most diffusive limiter inside the Sweby region; it follows the lower bound of it:

$\varphi(r)=\max (0, \min (1, r))$.

On the other hand, the superbee limiter, given by

$\varphi(r)=\max (0, \max (\min (2 r, 1), \min (r, 2)))$,

follows the upper bound of the Sweby region and is the most compressive limiter.

A well-known class of schemes is formed by the van Leer $\kappa$ schemes [30], which correspond to $\varphi(r)=\frac{1-\kappa}{2}+\frac{1+\kappa}{2} r, \kappa \in[-1,1]$. They are second-order accurate, and for $\kappa=\frac{1}{3}$ third-order accurate, but not monotone. The Koren limiter [18] follows the $\kappa=\frac{1}{3}$ scheme as much as possible inside the second-order Sweby region. It reads:

$\varphi(r)= \begin{cases}0, & r \leq 0 \\ \min \left(2 r, \min \left(\frac{1}{3}+\frac{2}{3} r, 2\right)\right), & r>0\end{cases}$ 


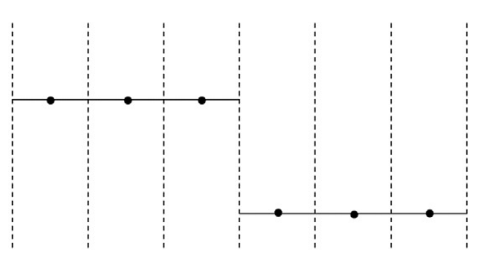

(a) before spatial reconstruction

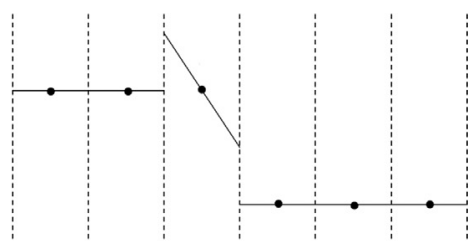

(b) after spatial reconstruction

Fig. 4. The values for a state variable in a number of cells. Here, application of spatial reconstruction causes an overshoot, due to a slope being chosen too large.

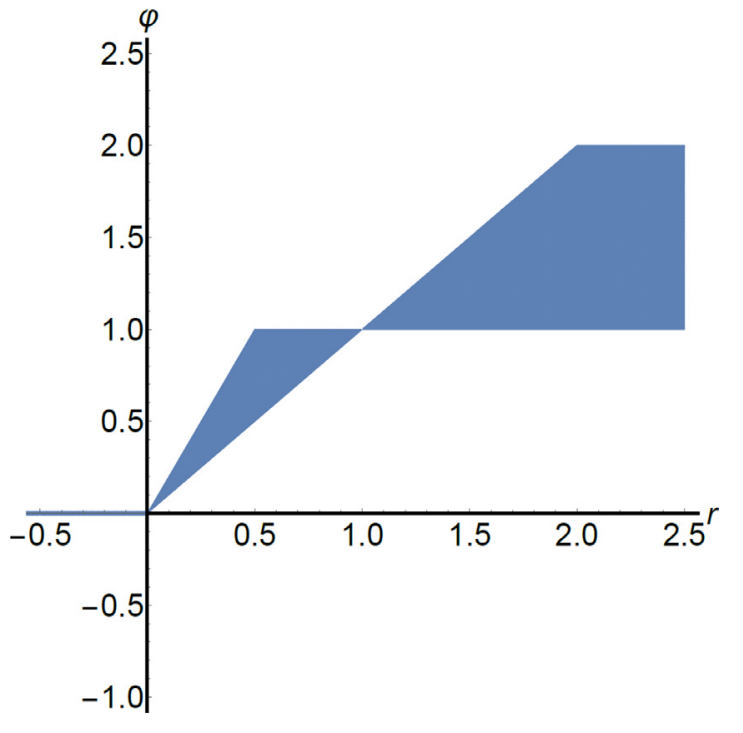

Fig. 5. The second-order accurate Sweby region.

Here we propose an extended $\kappa=\frac{1}{3}$-limiter, which for a range of negative $r$-values around $r=-1$ coincides with $\kappa=\frac{1}{3}$ as well:

$\varphi(r)= \begin{cases}\min \left(0, \max \left(-\frac{2}{3}, \frac{1}{3}+\frac{2}{3} r\right)\right), & r \leq 0, \\ \min \left(\frac{4}{3} r, \min \left(\frac{1}{3}+\frac{2}{3} r, 2\right)\right), & r>0 .\end{cases}$

As illustrated in Fig. 6, the newly proposed limiter function falls within the Spekreijse region with $M=2$. Therefore, following Eq. (24), using the limiter requires a time step with a CFL-number of at most $\frac{1}{2}$.

To illustrate the effect of the limiters, we compare them for a simple linear advection equation. The initial condition in Fig. 7a is a block profile, in 7b a sinusoid. Both move at constant speed. The domain has periodic boundary conditions, so the shape of the exact solution after $n$ periods, $(n=1,2, \ldots)$ is equal to the initial condition. We look at the numerical solutions after five periods, with 80 finite volumes. We see that all limiters result in a much sharper resolution of the traveling discontinuities, compared to the first-order method. The minmod limiter is indeed the most diffusive, whereas the superbee limiter the most compressive, even overly compressive in Fig. 7a, flattening the smooth gradient. The Koren limiter and the newly proposed one are in between these two extremes.

To examine the accuracy behavior of the new limiter, we perform an experimental error analysis. We numerically simulate the test case from Fig. 7a for 10,20,40,80,160 and 320 finite volumes. The time step size remains the same for all grid sizes. The time step is sufficiently small, such that for all grids, the temporal error is negligible with respect to the spatial error. The errors are determined by comparing the numerical results to the exact solution with the 1-norm. The results, in Fig. 8, show that both the Koren limiter and the newly proposed limiter perform between second and third order accurately.

\subsection{HLLC Riemann solver}

To calculate the fluxes in Eq. (16), we apply an HLLC-type solver to the homogeneous system, dimension by dimension. The next steps will be described in the following sections. Whereas the equations are in terms of conservative variables, the flux computation through the approximate Riemann solver is based on the primitive variables $\rho, u, v, p, \beta, \alpha$. Using the equations of state (8a) and (8b) we convert one set of variables into the other. The fluxes $F$ are determined using an HLLC-type method. We will present this method for the $x$-direction, the $y$-direction is treated analogously.

For the current system of equations, the HLLC scheme assumes four regions of constant states separated by three wave fronts, see Fig. 9. This boils down to the assumption that the outer two are shock waves. The resolution of shock waves is therefore exact. The middle wave front, a contact discontinuity, is also numerically exact. The middle two states are referred to as star regions, and we will denote the variables within these regions with the superscript *. The outer two states are taken as the primitive variables resulting from the MUSCL method on either side of the cell face considered. We distinguish them with subscripts $L$ and $R$.

Various estimates for the wave speeds have been suggested in the literature [29]. Care must be taken when selecting one, because the various estimates described in the literature differ a lot, affecting the numerical scheme, and because they are defined through the equation of state, with the speed of sound [3]. We will work with the following estimates for the wave speed of the three fronts [29]:

$S_{L}=\min \left(u_{L}-c_{L},(u-c)^{\mathrm{Roe}}\right)$,

$S_{R}=\max \left(u_{R}+c_{R},(u+c)^{\mathrm{Roe}}\right)$,

$S_{M}=\frac{p_{R}-p_{L}+\rho_{L} u_{L}\left(S_{L}-u_{L}\right)-\rho_{R} u_{R}\left(S_{R}-u_{R}\right)}{\rho_{L}\left(S_{L}-u_{L}\right)-\rho_{R}\left(S_{R}-u_{R}\right)}$.

Here, the superscript Roe denotes Roe averaging, which is defined through the density, velocity and enthalpy $\left(H=E+\frac{p}{\rho}\right)$ :

$\rho^{\text {Roe }}=\sqrt{\rho_{L} \rho_{R}}$

$u^{\text {Roe }}=\frac{\sqrt{\rho_{L}} u_{L}+\sqrt{\rho_{R}} u_{R}}{\sqrt{\rho_{L}}+\sqrt{\rho_{R}}}$,

$H^{\text {Roe }}=\frac{\sqrt{\rho_{L}} H_{L}+\sqrt{\rho_{R}} H_{R}}{\sqrt{\rho_{L}}+\sqrt{\rho_{R}}}$.

Furthermore, following the model's underlying assumptions, Eq. (3), we impose the following conditions:

$u_{L}^{*}=u_{R}^{*}=S_{M}$,

$p_{L}^{*}=p_{R}^{*}=p^{*}$ 

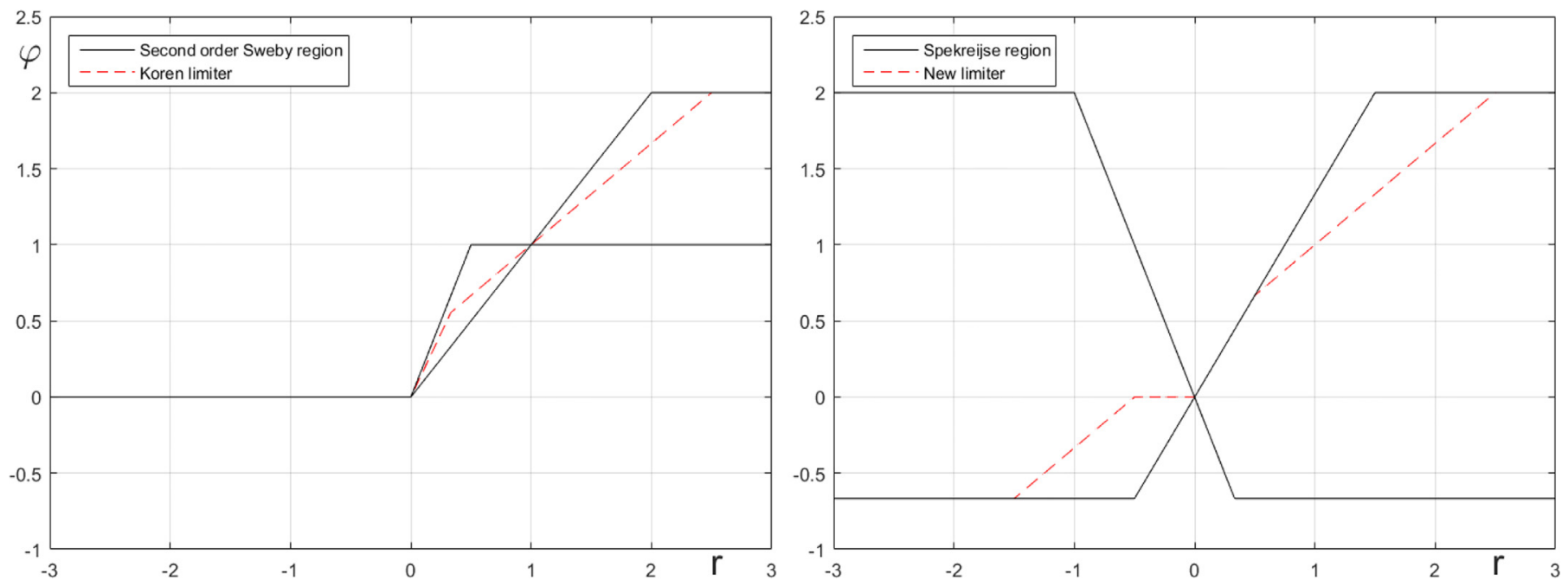

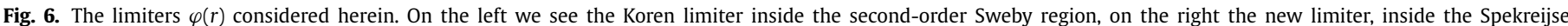
region for $M=2$ and $m=-\frac{2}{3}$.

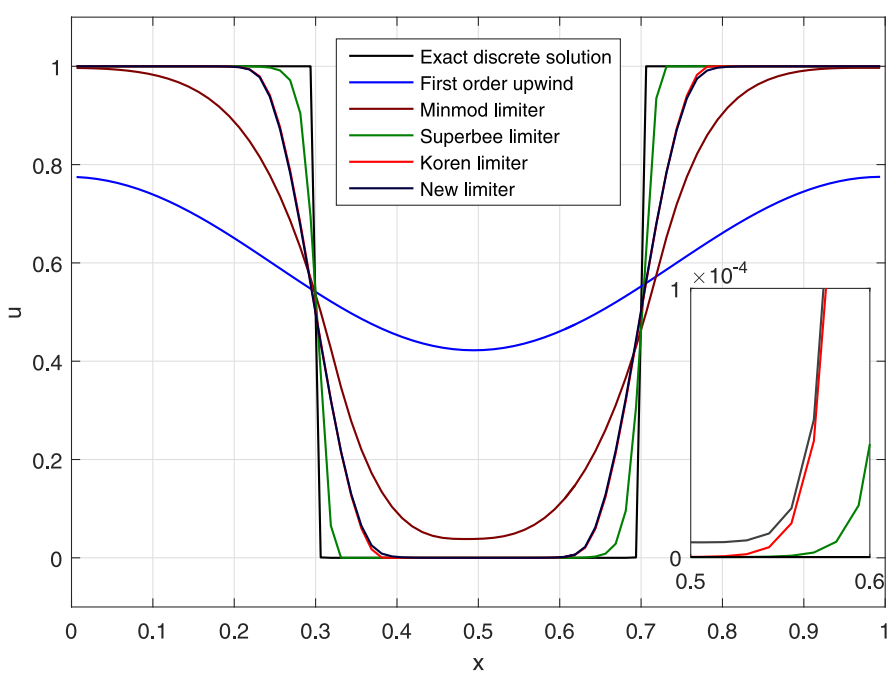

(a)

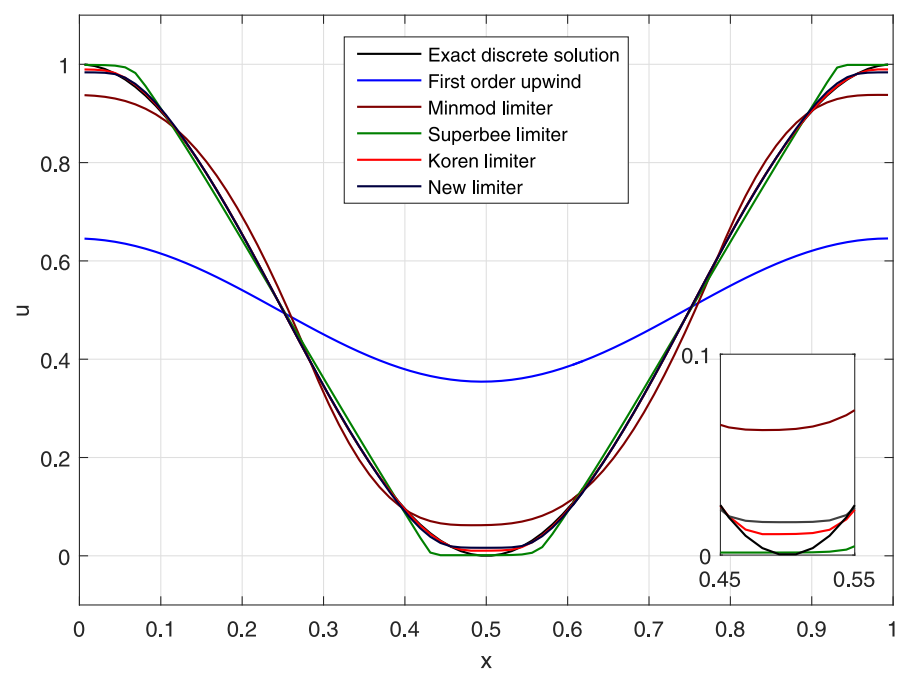

(b)

Fig. 7. Comparison of various limiters and their effect on the linear advection, over 5 periods, of a discontinuous (a) and an infinitely smooth (b) initial condition.

To obtain the quantities in the star region we consider the Rankine-Hugoniot jump conditions across the outer two wave fronts, assuming these are both shock waves:

$$
S_{K} \mathbf{Q}_{K}^{*}-\mathbf{F}\left(\mathbf{Q}_{K}^{*}\right)=S_{K} \mathbf{Q}_{K}-\mathbf{F}\left(\mathbf{Q}_{K}\right),
$$

$$
\begin{aligned}
S_{K}\left[\begin{array}{c}
\rho_{K}^{*} \\
\rho_{K}^{*} S_{M} \\
\rho_{K}^{*} v_{K}^{*} \\
\rho_{K}^{*} E_{K}^{*} \\
\alpha_{K}^{*} \rho_{1 K}^{*} \\
\alpha_{K}^{*}
\end{array}\right]- & {\left[\begin{array}{c}
\rho_{K}^{*} S_{M} \\
\rho_{K}^{*} S_{M}^{2}+p^{*} \\
\rho_{K}^{*} S_{M} v_{K}^{*} \\
S_{M}\left(\rho_{K}^{*} E_{K}^{*}+p^{*}\right) \\
\alpha_{K}^{*} \rho_{1 K}^{*} S_{M} \\
S_{M} \alpha_{K}^{*}
\end{array}\right]=S_{K}\left[\begin{array}{c}
\rho_{K} \\
\rho_{K} u_{K} \\
\rho_{K} v_{K} \\
\rho_{K} E_{K} \\
\alpha_{K} \rho_{1 K} \\
\alpha_{K}
\end{array}\right] } \\
& -\left[\begin{array}{c}
\rho_{K} u_{K} \\
\rho_{K} u_{K}^{2}+p_{K} \\
\rho_{K} u_{K} v_{K} \\
u_{K}\left(\rho_{K} E_{K}+p_{K}\right) \\
\alpha_{K} \rho_{1 K} u_{K} \\
u_{K} \alpha_{K}
\end{array}\right],
\end{aligned}
$$

with $K=L, R$. The equations are solved algebraically in a straightforward manner to obtain:

$\rho_{K}^{*}=\rho_{K} \frac{S_{K}-u_{K}}{S_{K}-S_{M}}$,

$v_{K}^{*}=v_{K}$,

$p^{*}=p_{K}+\rho_{K}\left(S_{M}-u_{K}\right)\left(S_{K}-u_{K}\right)$,

$E_{K}^{*}=E_{K}+\left(S_{M}-u_{K}\right)\left(S_{M}+\frac{p_{K}}{\rho_{K}\left(S_{K}-u_{K}\right)}\right)$,

$\alpha_{K}^{*} \rho_{1 K}^{*}=\alpha_{K} \rho_{1 K} \frac{S_{K}-u_{K}}{S_{K}-S_{M}}$,

$\alpha_{K}^{*}=\alpha_{K} \frac{S_{K}-u_{K}}{S_{K}-S_{M}}$.

Next we determine the flux in $x=0$, at the cell interface. We define four fluxes. The choice of the final flux is based on the sign 


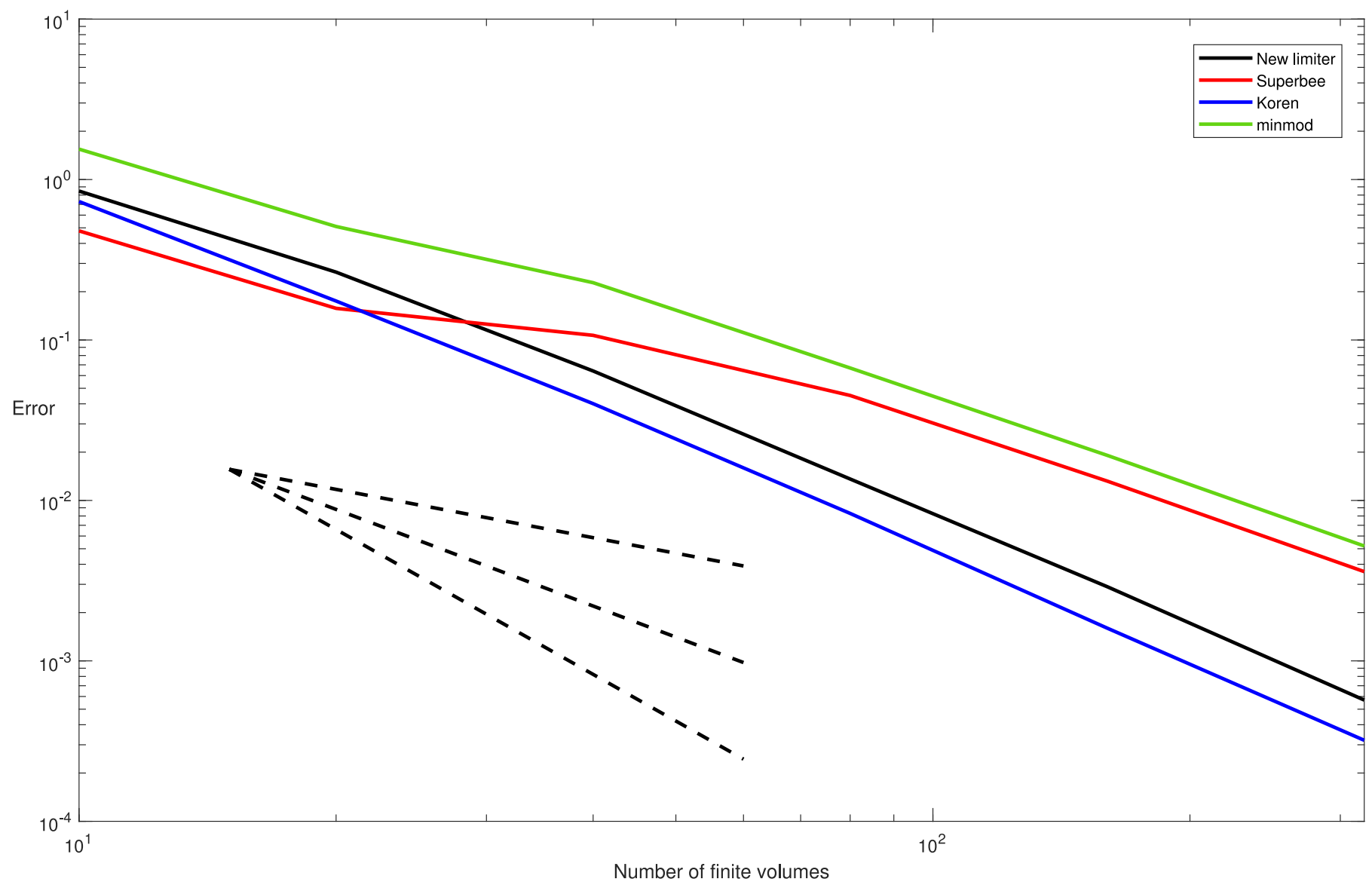

Fig. 8. Error analysis for the smooth initial condition from Fig. 7b, for various limiter functions (first-, second- and third-order accuracy indicated by dashed lines).

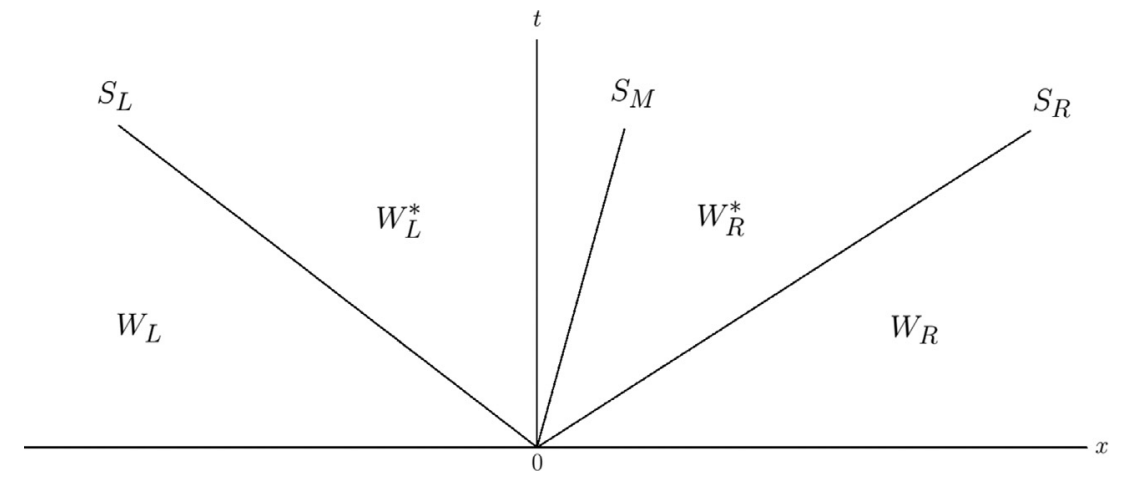

Fig. 9. The regions used in the HLLC solver.

of the three wave speed estimates:

$$
\mathbf{F}= \begin{cases}\mathbf{F}_{1}, & S_{L}, S_{M}, S_{R} \geq 0 \\ \mathbf{F}_{2}, & S_{R}, S_{M} \geq 0, S_{L}<0 \\ \mathbf{F}_{3}, & S_{R} \geq 0, S_{L}, S_{M}<0 \\ \mathbf{F}_{4}, & S_{L}, S_{M}, S_{R}<0\end{cases}
$$

with

$$
\mathbf{F}_{1,4}=\left[\begin{array}{c}
\rho_{K} u_{K} \\
\rho_{K} u_{K}^{2}+p_{K} \\
\rho_{K} u_{K} v_{K} \\
u_{K}\left(\rho_{K} E_{K}+p_{K}\right) \\
\rho_{1 K} \alpha_{K} u_{K} \\
\alpha_{K} u_{K}
\end{array}\right], \quad K=L, R \text { respectively, }
$$

and

$$
\mathbf{F}_{2}=\mathbf{F}_{1}+S_{L}\left(\mathbf{Q}_{L}^{*}-\mathbf{Q}_{L}\right), \quad \mathbf{F}_{3}=\mathbf{F}_{4}+S_{R}\left(\mathbf{Q}_{R}^{*}-\mathbf{Q}_{R}\right)
$$

The flux $\mathbf{F}$ is calculated for every cell interface and complemented with fluxes at the edges of the domain resulting from the boundary conditions.

\subsection{Non-conservative volume fraction term}

The non-conservative term in Eq. (15), the right-hand side of the volume fraction advection Eq. (6), is handled in an HLLC-type way as well, in the way proposed in [16]. In the $x$-direction this is 
done by:

$$
\begin{aligned}
& \mathbf{s}_{x, i}^{n}=\left(\alpha_{i}-\phi_{i}\right) \frac{1}{\Delta x}\left(\left(\begin{array}{ll}
u_{L}, & S_{L}, S_{M}, S_{R} \geq 0 \\
\frac{S_{L}-u_{L}}{S_{L}-S_{M}} S_{M}, & S_{R}, S_{M} \geq 0, S_{L}<0 \\
\frac{S_{R}-u_{R}}{S_{R}-S_{M}}, & S_{R} \geq 0, S_{L}, S_{M}<0 \\
u_{R}, & S_{L}, S_{M}, S_{R}<0
\end{array}\right)_{i+\frac{1}{2}}\right.
\end{aligned}
$$

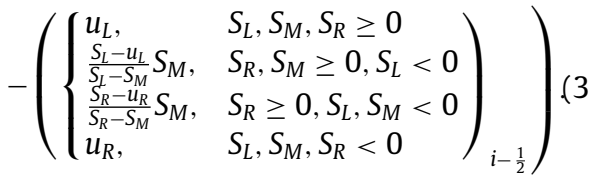

The $y$-direction is handled analogously.

\subsection{Positivity of the volume fraction}

With the combination of the non-conservative term in the equations, and the MUSCL method, it is not guaranteed that the volume fraction remains positive in the simulation. Since the volume fraction of fluid 2 must also be positive, the volume fraction itself must remain in the [0,1]-interval. When applying the scheme to initial conditions with volume fractions of 0 and 1 , the volume fraction may lose positivity within several time steps. To prevent this, the volume fractions in the initial conditions are changed into $\varepsilon$ and $1-\varepsilon$, for some small value of $\varepsilon$. The mass fractions are changed accordingly. Numerical tests have shown that the choice of limiter has a major influence on the value of $\varepsilon$ which needs to be chosen. The newly proposed limiter allows a value of $\varepsilon=10^{-10}$, whereas the other limiter functions considered in this work require an $\varepsilon$ of $\mathcal{O}\left(10^{-5}\right)$. Because of the large density ratio between the two fluids, which in the case of a liquid and a gas could easily be $\mathcal{O}\left(10^{3}\right)$, this could result in an error of $\mathcal{O}\left(10^{-2}\right)$ in the initial condition, which will propagate throughout the numerical simulation. The newly proposed limiter clearly performs better for our purposes. The main reason that it performs better is to be sought in its negative values for negative $r$, corresponding to extrema. The extrema of the volume fraction are restrained more strongly. At the same time, the new limiter is third-order accurate in smooth regions.

\subsection{Time integration}

We implement an explicit third-order accurate Runge-Kutta method, in line with the spatial discretization, which is locally third-order accurate in smooth regions. The time integration method is described by the Butcher tableau [15]

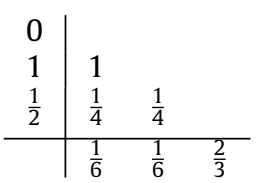

This time integrator has been outlined in [25], where it was shown to be a TVD Runge-Kutta method [20], meaning that the TVD property of the explicit Euler method extends to this time integrator.

\subsection{Boundary condition treatment}

Two types of boundary conditions are considered herein; one representing a solid wall, the other outflow. The numerical implementation of this is done by adding virtual cells around the edges of the domain. Then, a Riemann problem is solved, as is done in the interior cells, to determine the fluxes.

In the case of an outflow boundary the state in the virtual cell is copied from that of the adjacent interior cell. This lets waves run out of the computational domain as if the domain extends further.
The resulting Riemann problem is trivial; $F\left(q_{L}, q_{r}\right)$ with $q_{L}=q_{R}=q$, hence $F(q, q)=f(q)$.

The solid wall is modeled by reflection; the state in the adjacent cell is copied, with the exception of the velocity in the direction perpendicular to the cell boundary, which changes sign. This results in a Riemann problem with either two identical shock waves or two identical rarefaction waves. The middle wave has speed zero. The only nonzero flux in the conservative part of the equations is in the momentum equation, due to the pressure.

The non-conservative part of the flux is determined in the same way as for the cell boundaries in the interior.

\subsection{Gravity forces}

In the next section we will consider a liquid column, driven by a gravity force $g$. This is modeled by adding a source term $\mathbf{s}_{g}$ to the system (7a), in one dimension:

$\frac{\partial}{\partial t} \mathbf{q}+\frac{\partial}{\partial x} \mathbf{f}=\mathbf{s}+\mathbf{s}_{g}$

with

$\mathbf{q}=\left[\begin{array}{c}\rho \\ \rho u \\ \rho E \\ \alpha \rho_{1} \\ \alpha\end{array}\right], \mathbf{f}=\left[\begin{array}{c}\rho u \\ \rho u^{2}+p \\ u(\rho E+p) \\ \alpha \rho_{1} u \\ \alpha u\end{array}\right], \mathbf{s}=\left[\begin{array}{c}0 \\ 0 \\ 0 \\ 0 \\ (\alpha-\phi)\left(u_{x}\right)\end{array}\right]$,

$\mathbf{s}_{g}=\left[\begin{array}{c}0 \\ \rho g \\ \rho g u \\ 0 \\ 0\end{array}\right]$.

This small addition to the model does not affect the underlying structure of the system. The additional source term due to gravity is handled separately. In numerical calculations of $\mathbf{s}_{g}$ we naturally take the cell averages for $\rho$ and $u$.

\section{Generalized Bagnold model}

In 1939, Bagnold pioneered research on wave impacts [2]. He provided a comprehensive overview of breaking and non-breaking free-surface waves, and performed experiments in which he studied the impact. He introduced a one-dimensional model of breaking wave impact with entrapped air to predict pressures.

In the original work of Bagnold [2] wave impacts are modeled by a solid piston with an initial velocity, moving towards a wall, with an ideal gas trapped in between, see also $[22,28]$. The problem then depends on only two parameters: the adiabatic constant of the gas, $\gamma_{g}$, and the dimensionless Bagnold number $S_{B}$, describing the magnitude of the impact.

We consider a generalized Bagnold model [6], which consists of a tube closed on both ends, containing an incompressible liquid in between two gas columns. The liquid piston is driven by a body force instantaneously applied at $t=0$, for instance gravity. The situation is illustrated in Fig. 10.

This situation is simulated using the computational method presented before. The numerical results are compared to analytical results which we outline in the following.

\subsection{Analytical solution}

The dimensionless formulation of the generalized Bagnold model is given briefly. For a more elaborate description, see [6]. We assume the wave impact process to be adiabatic and reversible. Furthermore, the liquid is assumed incompressible, and we therefore represent it as a solid piston. The compression in the gas is 


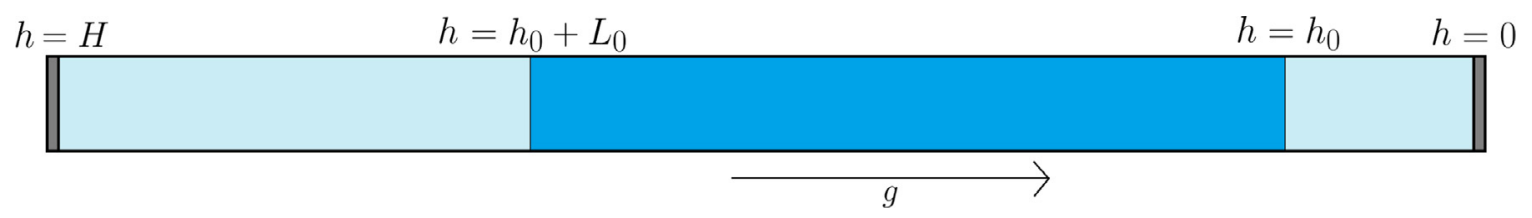

Fig. 10. The initial condition in the generalized Bagnold model.

Table 1

Description of the cases.

\begin{tabular}{lllllll}
\hline Case & Scale & Liquid & Gas & Temperature $\left({ }^{\circ} \mathrm{C}\right)$ & Pressure $(\mathrm{Pa})$ & Impact number \\
\hline 1 & $1: 1$ & LNG & NG & -162 & $10^{5}$ & 0.357 \\
2 & $1: 40$ & LNG & NG & -162 & $10^{5}$ & 0.00893 \\
3 & $1: 40$ & Water & Air & 20 & $10^{5}$ & 0.0196 \\
4 & $1: 40$ & Water & $\mathrm{SF}_{6}+\mathrm{N}_{2}$ & 20 & $10^{5}$ & 0.0196 \\
5 & $1: 40$ & $1: 40$-scaled LNG & $1: 40$-scaled NG & -162 & 2500 & 0.357 \\
\hline
\end{tabular}

uniform. The solid piston model results in the following equation for the height of the gas pocket at time $t$ :

$h^{\prime \prime}(t)=-g-\frac{p_{0}}{\rho_{l} L_{0}}\left(\left(\frac{H-L_{0}-h_{0}}{H-L_{0}-h(t)}\right)^{\gamma_{g}}-\left(\frac{h_{0}}{h(t)}\right)^{\gamma_{g}}\right), \quad h(0)=h_{0}$.

Here, $g$ is the gravitational acceleration, $p_{0}$ is the pressure at $t=0$, and $\rho_{l}$ is the density of the liquid piston. The other parameters are related to the dimensions of the system and shown in Fig. 10. The velocity $h^{\prime}(t)$ can be calculated analytically [28]. The compression of the gas pocket is considered to be adiabatic:

$\frac{p}{\rho^{\gamma_{g}}}=$ constant.

Since the density is inversely proportional to the length of the gas pocket, Eqs. (38) and (39) are enough to determine the pressure in the gas pocket.

In this generalized model, the situation is completely described by four dimensionless numbers:

- length aspect ratio between the liquid column and the lower gas pocket,

- density ratio between the gas and the liquid,

- Bagnold number $S_{B}$, given by $S_{B}=\frac{L_{0} \rho_{0,1} g}{p_{0}}$,

- adiabatic constant of the gas $\gamma_{g}$.

In the numerical method the liquid is compressible, so a fifth dimensionless number is added to this list: liquid compressibility $\frac{p_{0}}{\rho_{0, l}\left(c_{0, l}\right)^{2}}$. If the model is scaled in such a way that these dimensionless quantities remain the same, the scaling is completely similar. Brosset et al. [6] discuss this model, with a non-zero initial velocity, and refer to the scaling as complete Froude scaling.

\subsection{Comparison of analytical and numerical results}

At the ISOPE conference in 2010, the simulation of the generalized Bagnold model was given as a benchmark study for wave impact simulation methods [12]. The participants were given five sets of initial data to be simulated, given in Table 1.

The scaling in the second column refers to Froude scaling. The 1:40 scaled LNG and NG refer to complete Froude scaling, in which the fluid properties are changed to obtain a complete similarity in the different scales between Cases 1 and 5, which should yield exactly the same results. The scaling results in fluids that are not physically meaningful, but provide valuable results anyhow. Furthermore the time of the numerical calculation can be decreased by scaling in this way, because the speed of sound of the fluids is affected by it.
Table 2

Fluid properties.

\begin{tabular}{llll}
\hline Fluid & $\gamma$ & $\rho_{0}\left(\mathrm{~kg} / \mathrm{m}^{3}\right)$ & $c(\mathrm{~m} / \mathrm{s})$ \\
\hline LNG $\left(-162{ }^{\circ} \mathrm{C}\right)$ & 15.35 & 455 & 1300 \\
Water $\left(20^{\circ} \mathrm{C}\right)$ & 7 & 1000 & 1500 \\
$1: 40$-scaled LNG & 15.35 & 455 & 206 \\
$\mathrm{NG}\left(-162{ }^{\circ} \mathrm{C}\right)$ & 1.3 & 1.82 & 267 \\
Air $\left(20^{\circ} \mathrm{C}\right)$ & 1.4 & 1.2 & 342 \\
$\mathrm{SF}_{6}+\mathrm{N}_{2}\left(20^{\circ} \mathrm{C}\right)$ & 1.13 & 4 & 168 \\
$1: 40$-scaled NG & 1.3 & 1.82 & 42 \\
\hline
\end{tabular}

Table 3

Lengths.

\begin{tabular}{ll}
\hline Length & $(\mathrm{m})$ \\
\hline$H$ & 15 \\
$L_{0}$ & 8 \\
$h_{0}$ & 2 \\
\hline
\end{tabular}

In all of these cases the compressibility of the liquid and the acoustics of the gas do not play a significant role. We expect the Kapila model and the piston model to produce the same results. The relevant properties of the fluids are given in Table 2 and the lengths are given in Table 3. These are the dimensions of the initial problem, Case 1. In the other cases, these dimensions are scaled down by a factor 40 .

Like in Dias et al. [12] we plot the pressures of all cases in one figure (Fig. 11). This requires a rescaling of the results according to complete Froude scaling, time and pressure are for instance rescaled by factors of $\sqrt{40}$ and 40 respectively. All numerical calculations are performed with 150 finite volumes and a CFL number of 0.4 (For the time integrator and limiter used, the upper bound for this CFL number, a TVD bound, is 0.5).

We note that the two-fluid model and the Bagnold model show an excellent agreement. Furthermore the complete Froude similarity of Cases 1 and 5 is demonstrated in Fig. 12.

The analytic solution assumes an incompressible liquid. The difference between this solution and our numerical solution, which includes liquid compressibility, is seen clearly when raising $g$ from $9.81 \mathrm{~m} / \mathrm{s}^{2}$ to for instance a 30 times larger value, thereby raising the impact number.

In Fig. 13 we see that the solutions from the two models diverge for increasing values of $g$. The liquid compressibility has the expected cushioning effect on the impact pressures. 


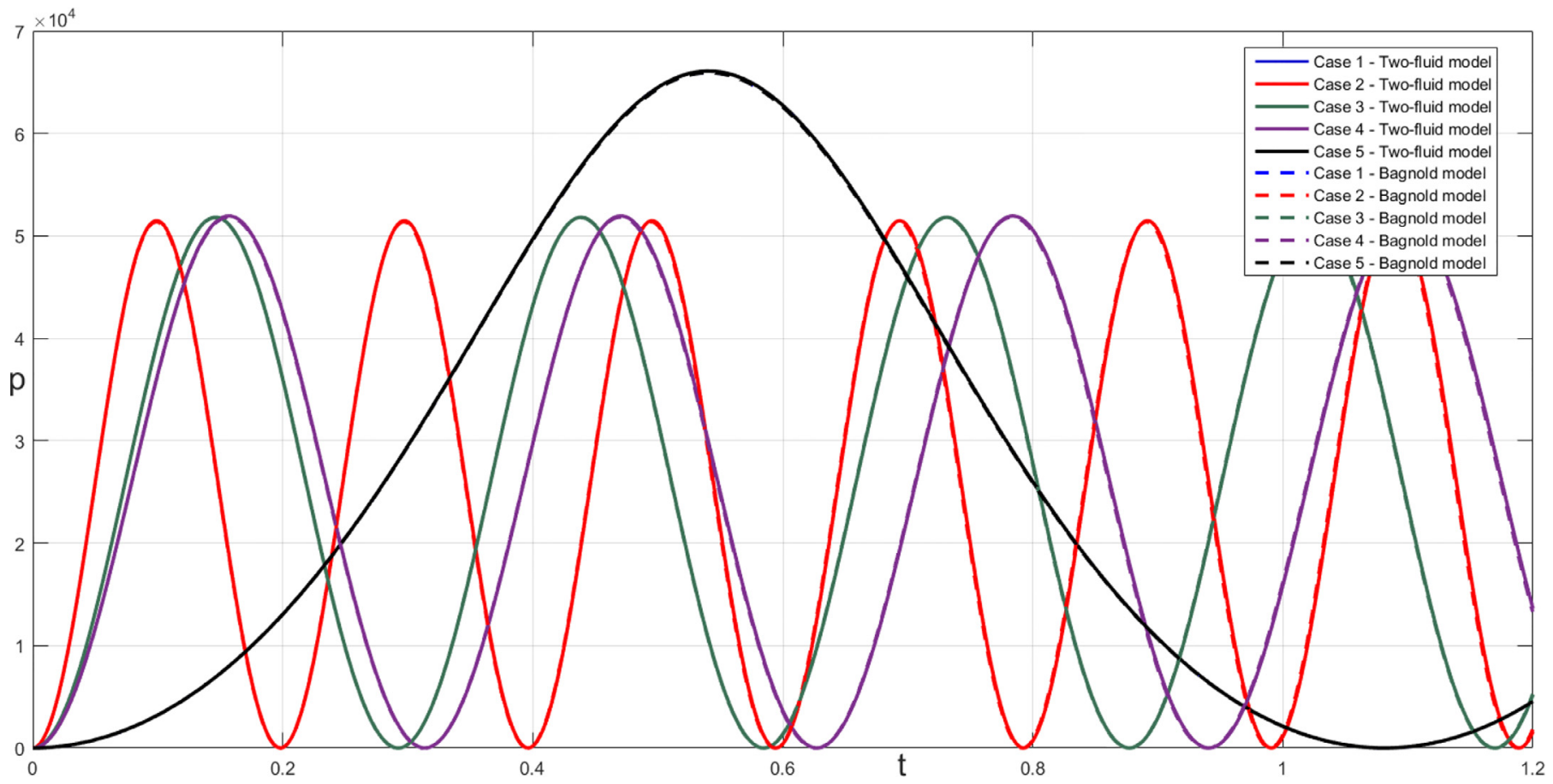

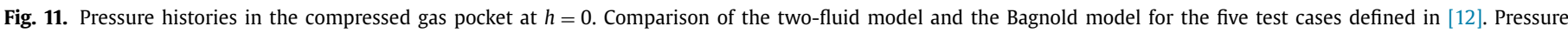
$p$ in Pascal and time $t$ in seconds..

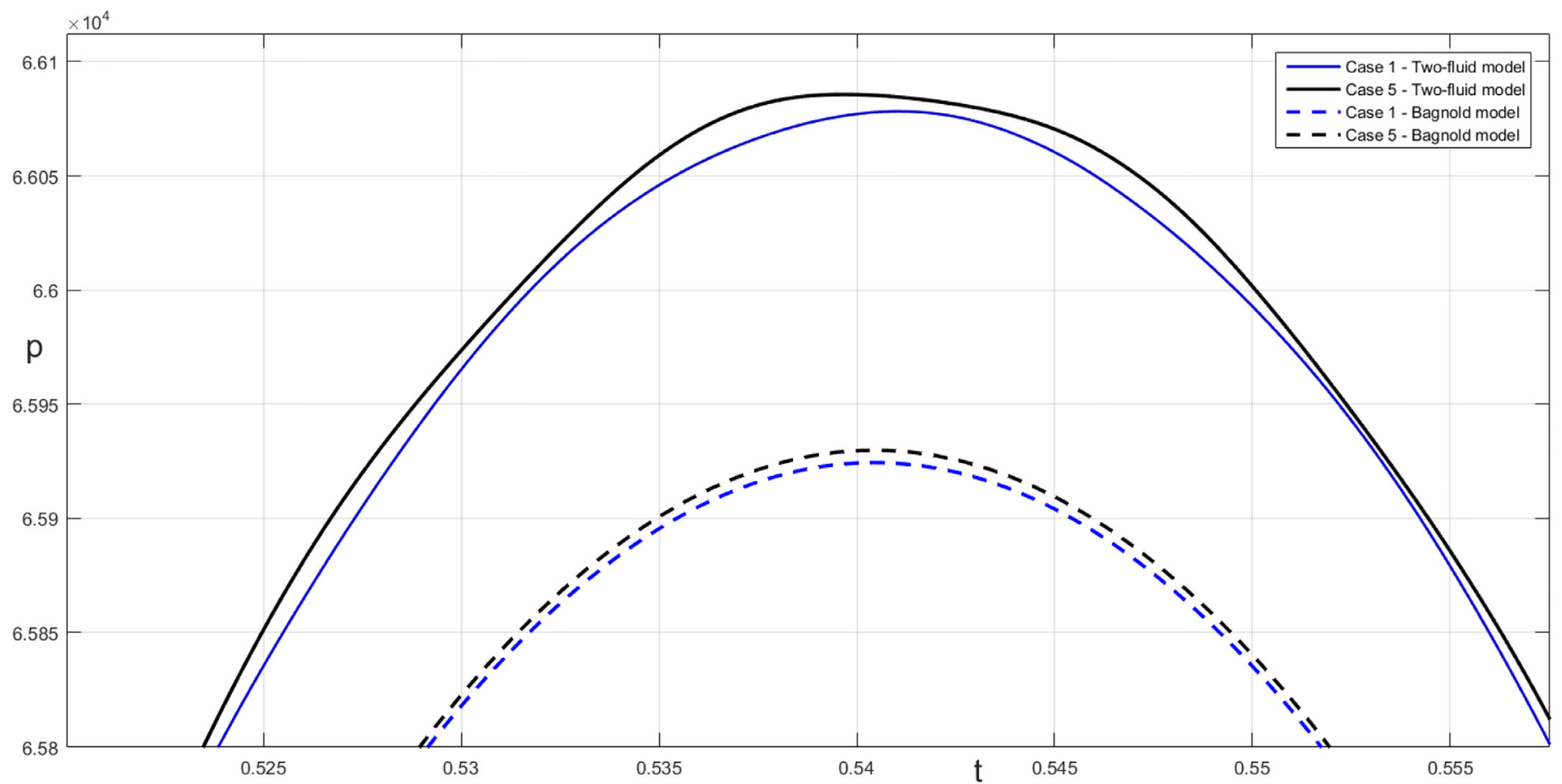

Fig. 12. Zoom in on Fig. 11. Pressure $p$ in Pascal and time $t$ in seconds..

\section{Shock-bubble interaction}

Haas and Sturtevant [14] performed experiments on shockbubble interaction. We consider in the experiments the results obtained with cylindrical bubbles, which we compare with our twodimensional numerical simulations.

The bubble is filled with a gas different from the surrounding air and contained within a thin film. Both the bubble and the surrounding air are at rest initially. A shock wave hits the cylindrical bubble from aside and interacts with it. The length and time scales involved are such that mixing of the fluids does not have a significant effect, making the two-fluid model suitable to describe the situation.

Two variants have been considered: one in which the bubble is filled with a heavier gas than the surrounding air (R22) and one with a lighter gas (helium, mixed with some air). The density of the gas has a major effect on the deformations the bubble undergoes. 


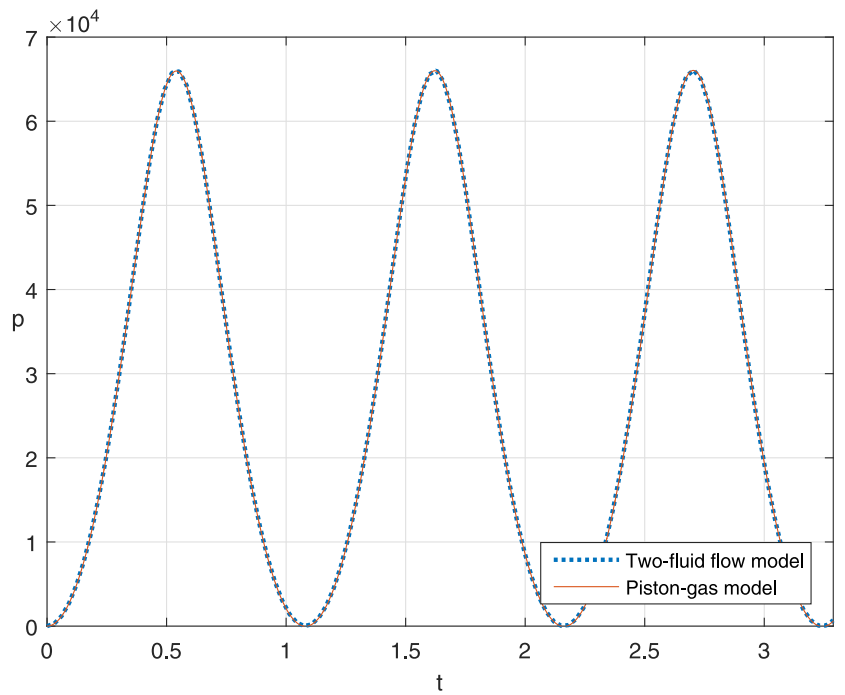

(a) $g$

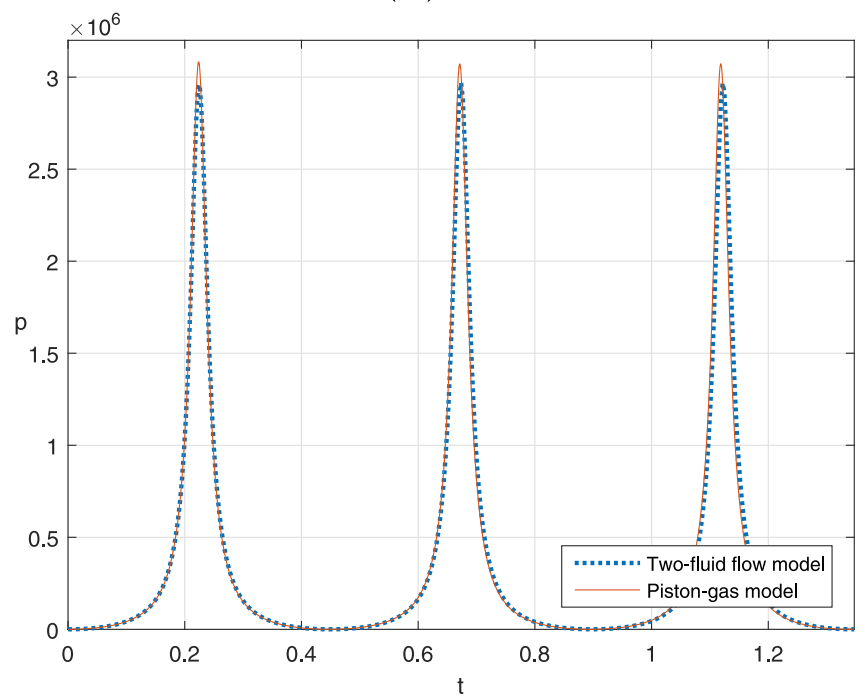

(c) $10 g$

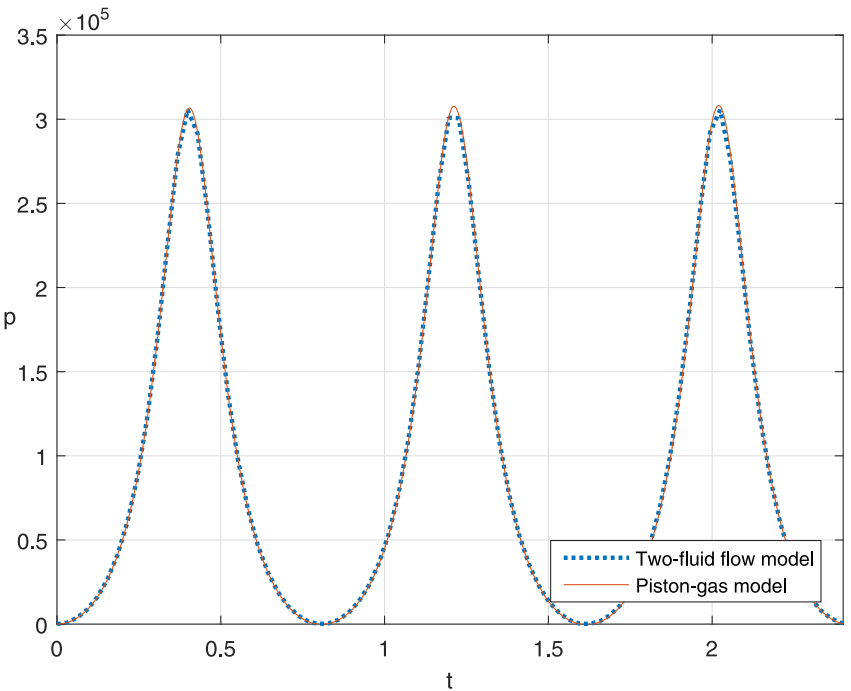

(b) $3 g$

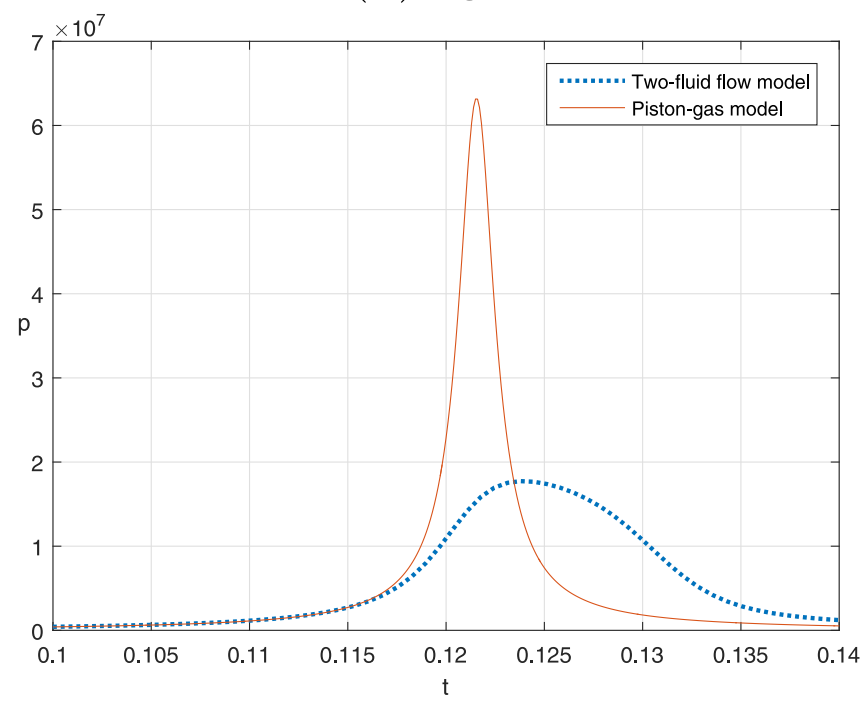

(d) $30 \mathrm{~g}$

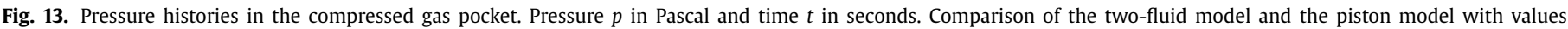
of $g$ : (a) for $g\left(=9.81 \mathrm{~m} / \mathrm{s}^{2}\right)$, (b) $3 g$, (c) $10 \mathrm{~g}$, (d) $30 \mathrm{~g}$. Due to liquid compressibility, the results from the two models diverge for higher values of $g$.

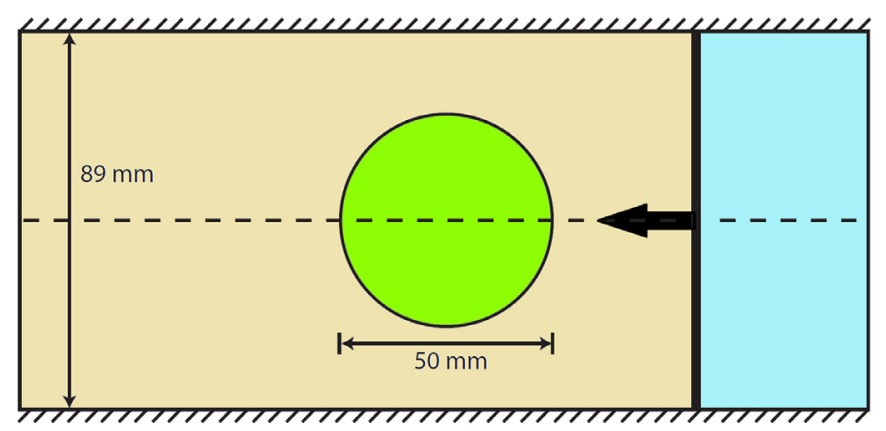

Fig. 14. Sketch of the initial condition of the Haas and Sturtevant experiment [14].

The experiment resulted in a number of shadow photographs which clearly show what happens to the bubble. This has become a benchmark for numerical simulation of compressible two-fluid flows $[19,21]$. In both cases, we numerically simulate the situa- tion on a $356 \times 960$ rectangular grid with the new limiter function and a CFL number of 0.24 . The limiters that we are using allow for a maximal CFL number of $\frac{1}{2}$ in one dimension. Simulating in two dimensions further decreases this CFL number with a factor 2 [29], yielding an upper bound for the CFL number of $\frac{1}{4}$. We use a CFL number slightly below this upper bound. Numerical tests have shown that this allows us to take the value of $\varepsilon$ introduced in Section 3.4 to be $10^{-10}$, whereas the other limiter functions considered require a value of $\varepsilon=10^{-5}$ to preserve positivity of the volume fraction. The symmetry in the $y$-direction is utilized to simulate only half of the domain, so symmetry in the flow is imposed.

\section{1. $R 22$}

For the case with a heavy gas in the bubble, R22, the density resulting from the numerical simulation and the shadow photographs from the experiment in [14] are juxtaposed. Note that the shadow photographs still show the initial position of the bubble, 

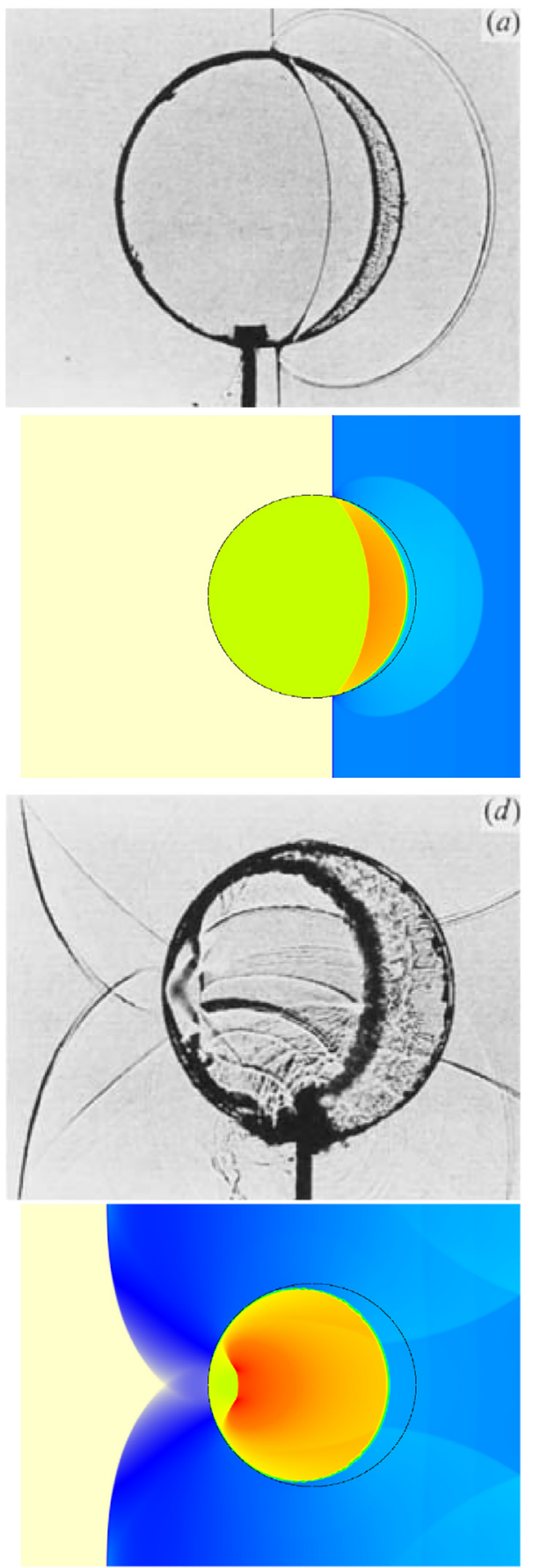

d)
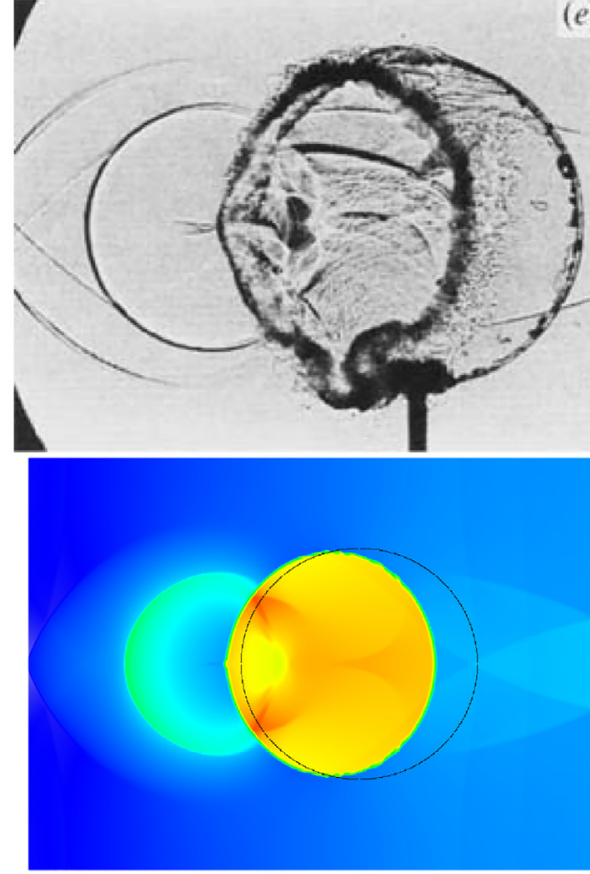

(b)
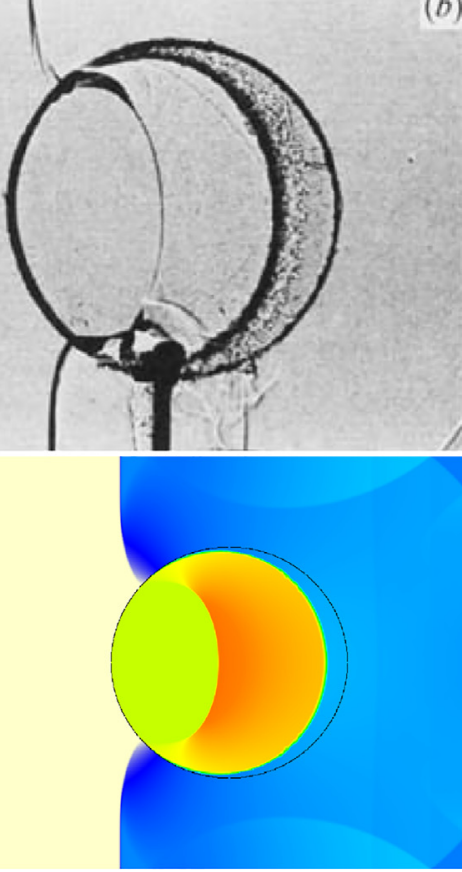

(e)

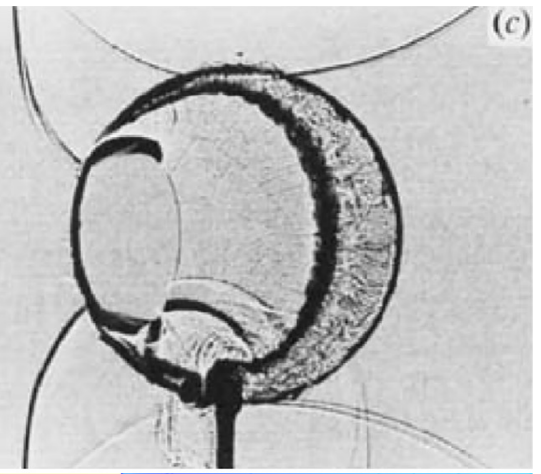

(c)
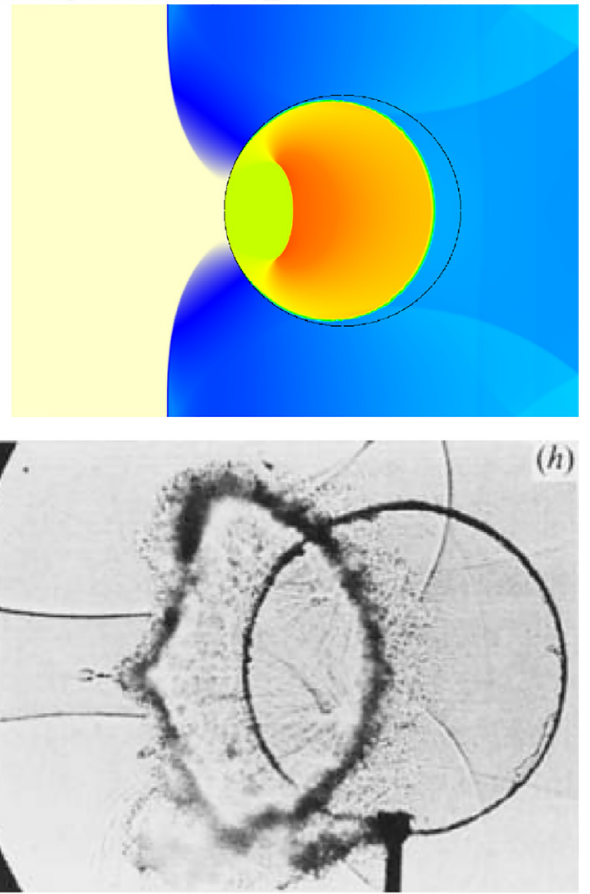

(h)

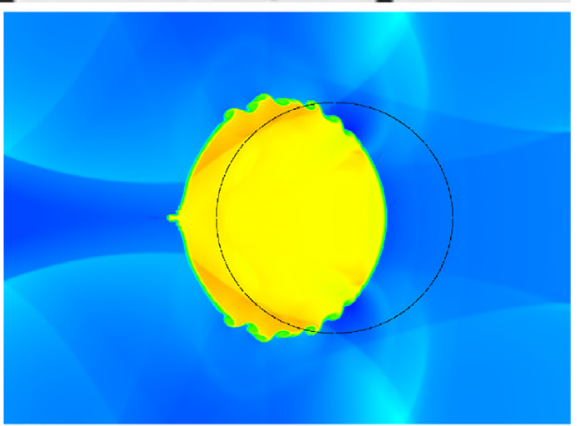

2

5

$\rho$

6

7

8

9

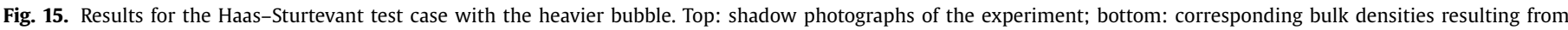
the numerical simulation. The color scheme in the plot from the simulation has been adjusted to highlight the important density gradients.

due to a remainder of the film. For a better comparison, we added this to the plots of the numerical results as well.

The speed of sound inside the bubble is lower than that of the surrounding air, making the shock move around the bubble faster than inside the bubble. The progressively darker colors inside the bubble indicate the coalescence of the refracted shocks, causing a strong rise in density. The bubble is initially compressed and later expands sideways. A good qualitative agreement is shown between the experiment and the numerical solution, especially in the outer region, where the wave speeds match and the same shock pattern is obtained. The numerical results show instabilities at the free surface, which in the experiment also occur and are RichtmyerMeshkov instabilities.

\subsection{Helium}

In the second case the bubble has a lower density than the surrounding air, so that the shock reaches its far side faster through 

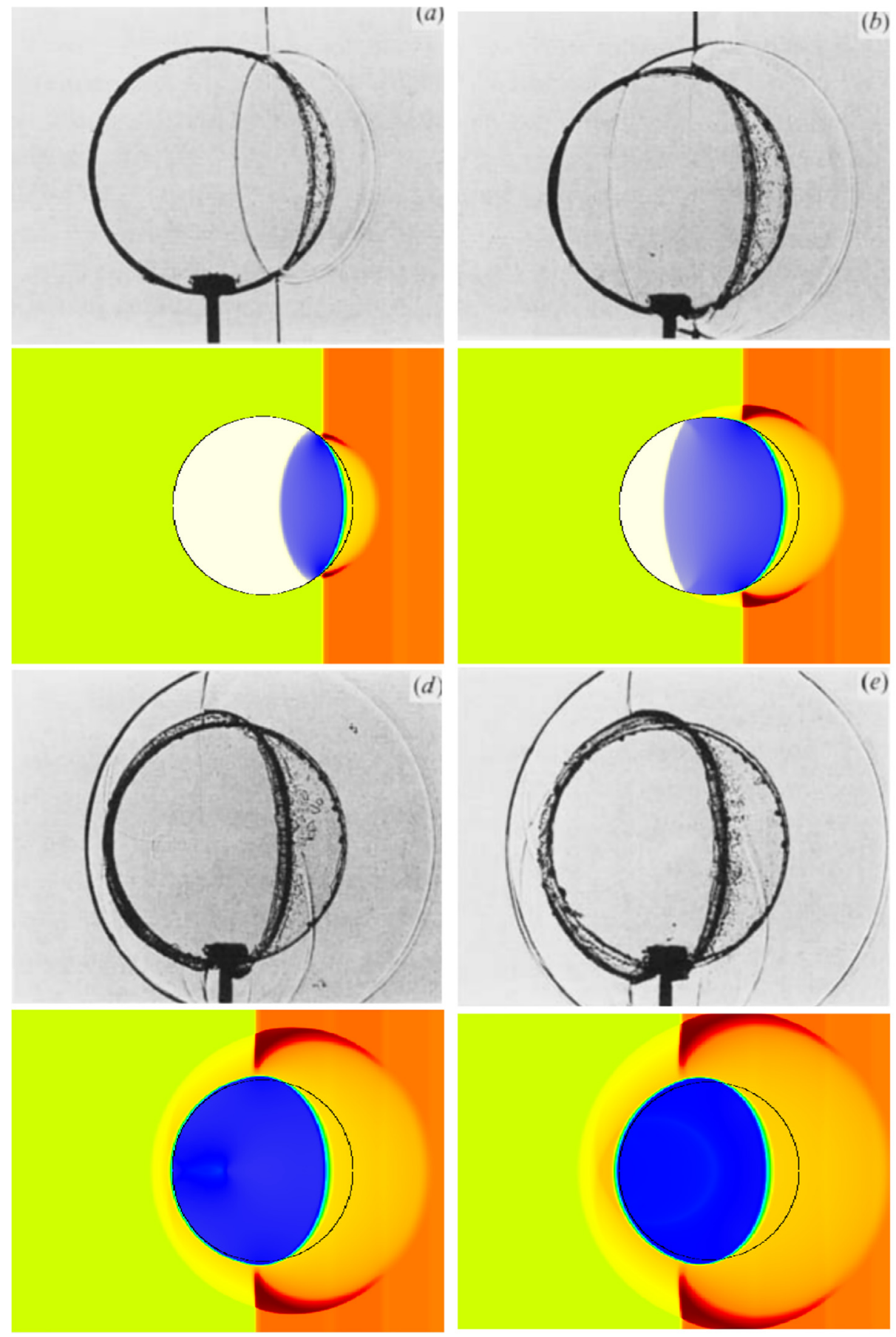

(b)
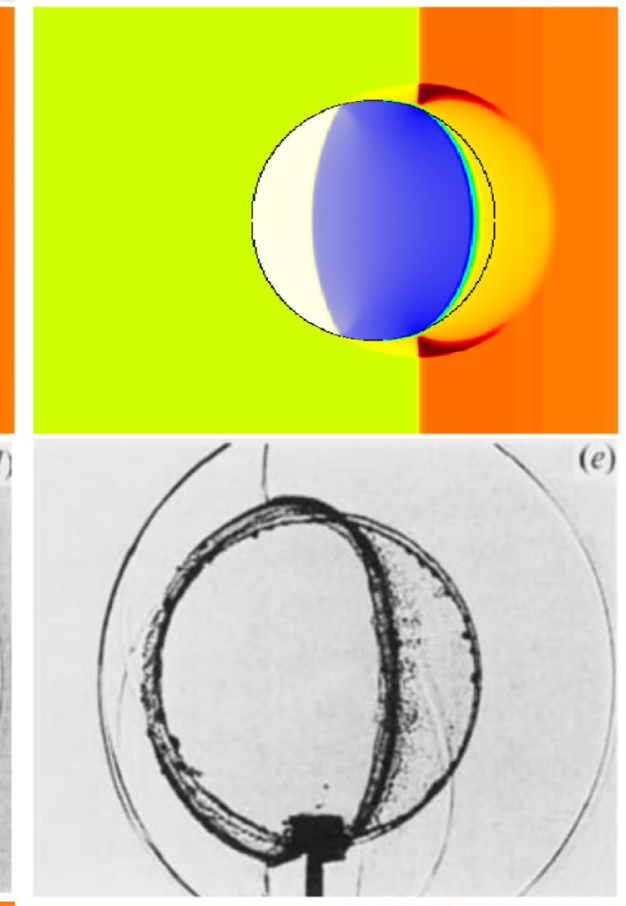

(e)
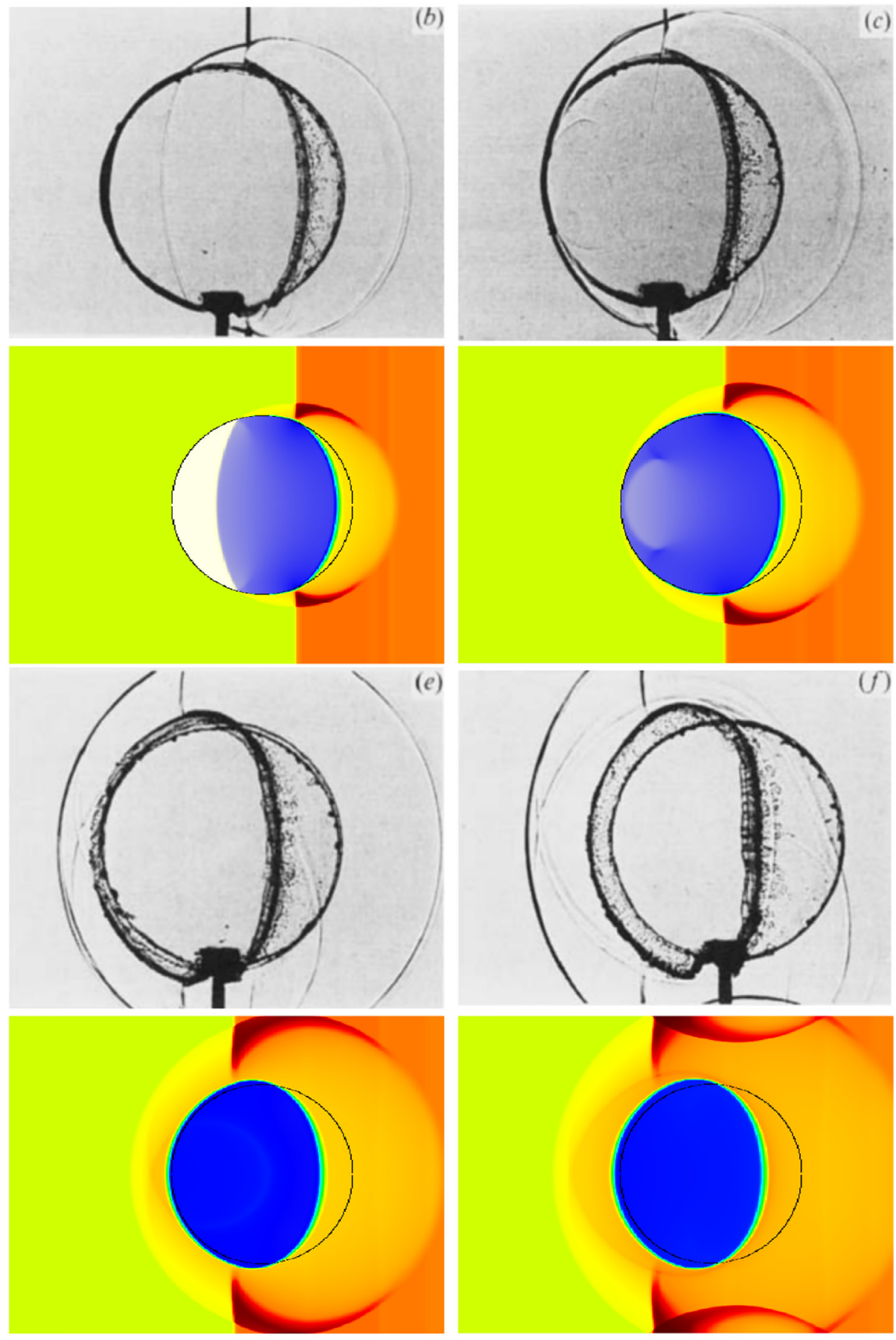

(c)
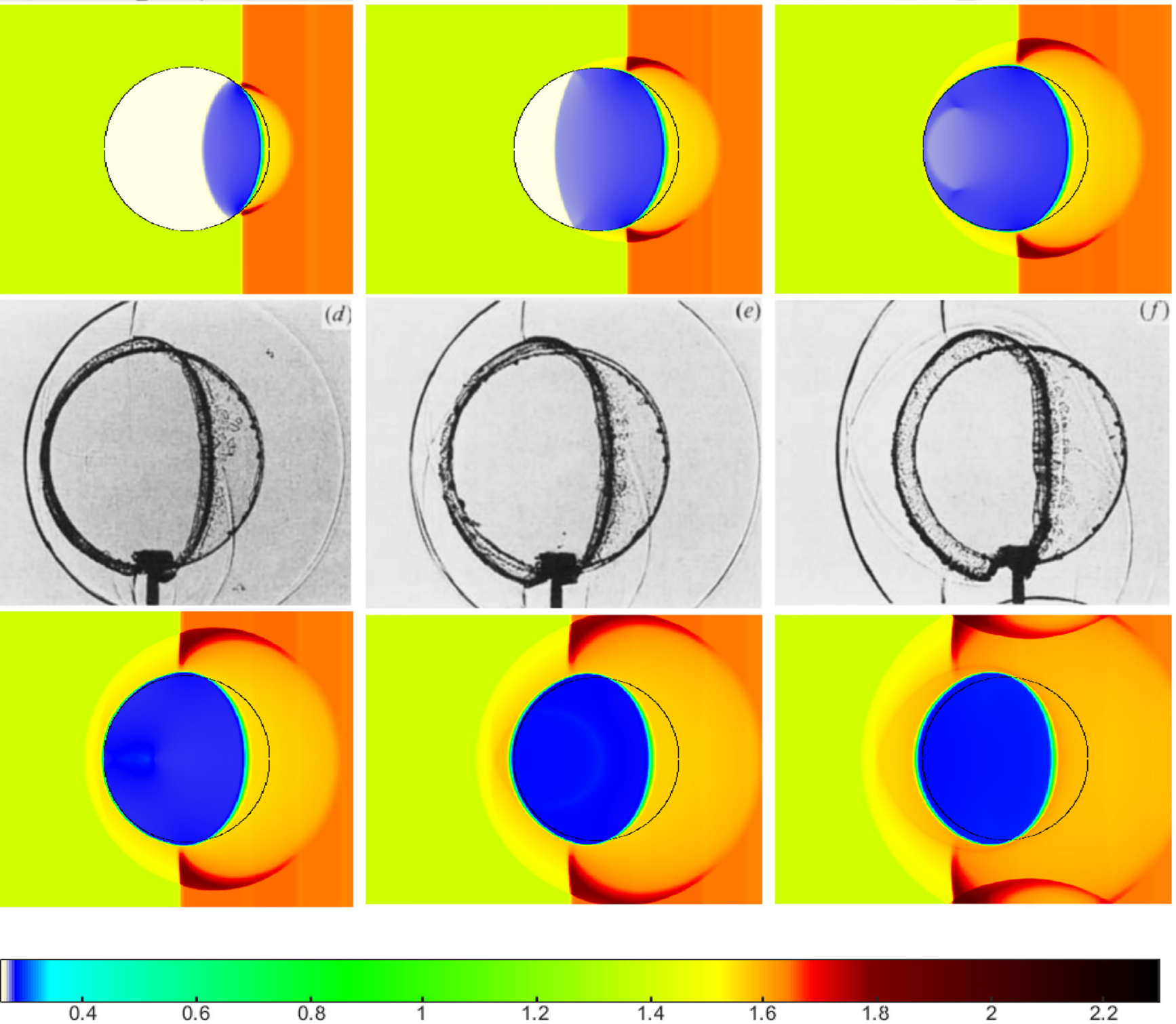

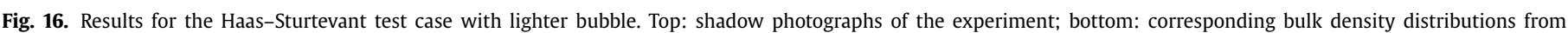
the numerical simulation. The color scheme in the plot from the simulation has been adjusted to highlight the important gradients.

the bubble than around it. The bubble compresses and later expands into a peanut-like shape. Eventually it splits up. Fig. 16 shows the numerical simulation together with the measurements. A good agreement is seen with regard to the propagation of the shock wave. The wave pattern, resulting from the interplay between the two fluids and the upper and lower walls is correctly captured as well.

\subsection{Importance of the parameter $\varepsilon$}

As indicated in Section 3.4, the parameter $\varepsilon$ is used to slightly change the initial condition, for positivity purposes. We take a low value for $\varepsilon\left(10^{-10}\right)$ and numerically simulate the R22 bubble again, for a longer time, for different limiter functions, all other parameters being the same. For this value of $\varepsilon$ the new limiter is able to 


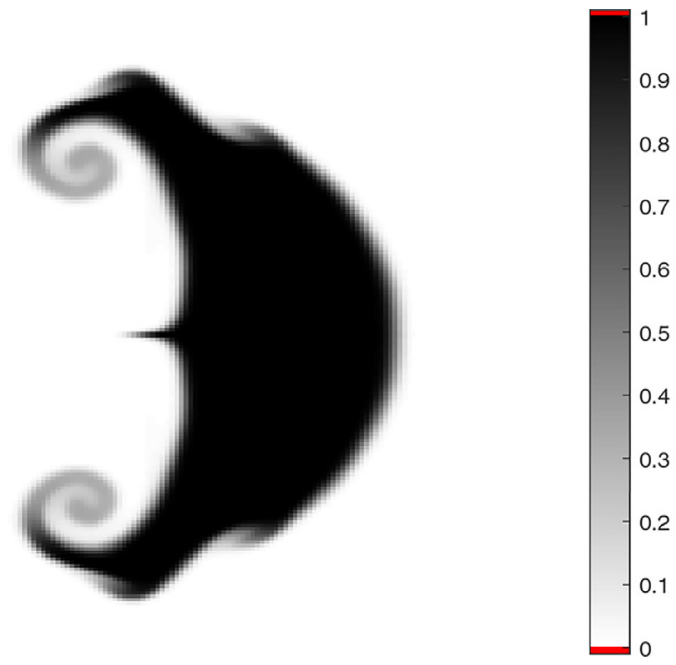

(a)
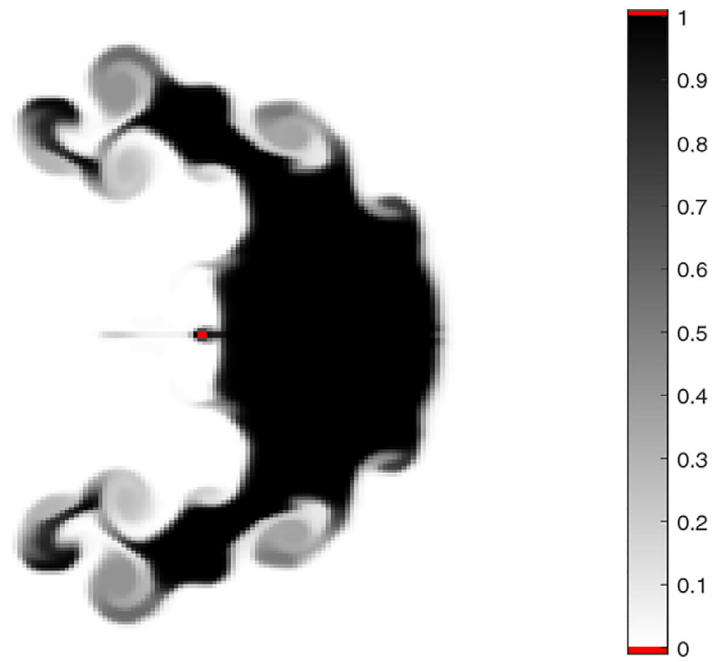

(b)

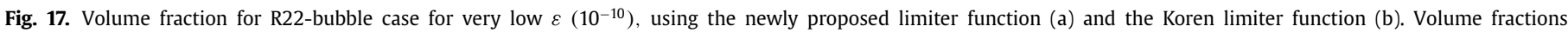

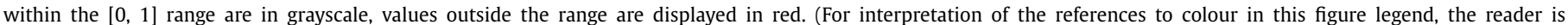
referred to the web version of this article.)

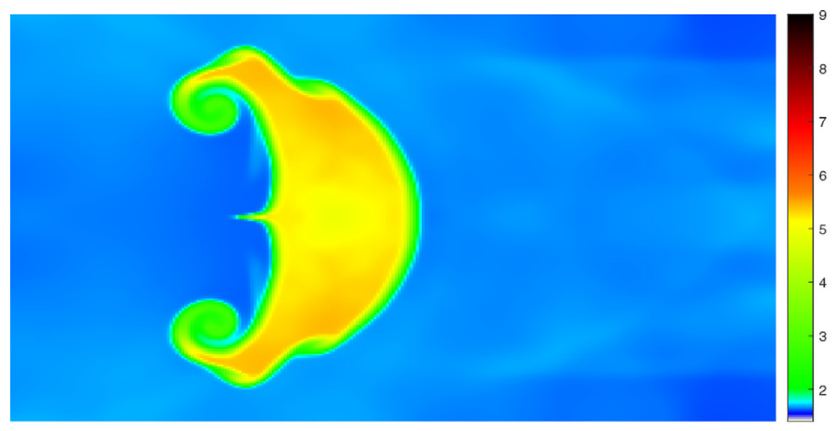

(a)

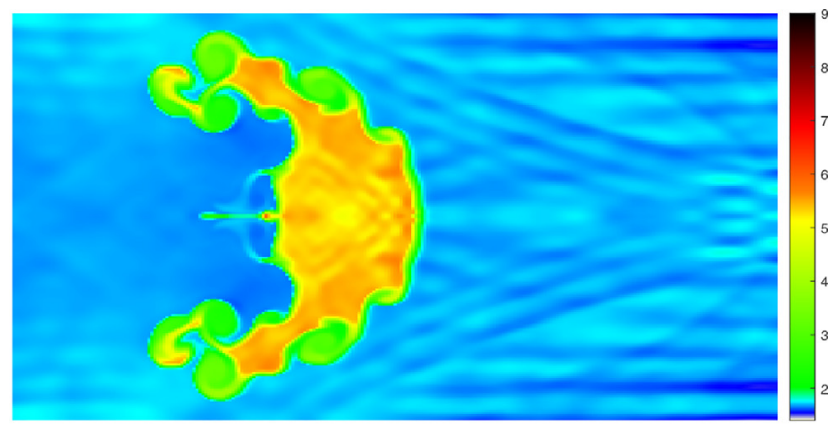

(b)

Fig. 18. Density for R22-bubble case for very low $\varepsilon\left(10^{-10}\right)$, using the newly proposed limiter function (a) and the Koren limiter function (b).

maintain positivity of the volume fraction, whereas the other limiter functions discussed in this paper are not. In Fig. 17, we compare results obtained with the new limiter and the Koren limiter. The new limiter result maintains positivity, whereas the Koren limiter locally does not. (For $\varepsilon=10^{-5}$, the Koren limiter yields nearly the same result as that in Fig. 17a.)

The corresponding density results are shown in Fig. 18. Even though the loss of positivity occurs very locally, it clearly has drastic effects. The spurious oscillations in the right half of Fig. 18b are a results.

\section{Conclusion}

In order to obtain a better understanding of the phenomenon of sloshing of LNG inside tanks, numerical simulations are required to supplement the data from experiments. To prepare for this, we considered a compressible two-fluid flow formulation, which uses the Kapila model.
A shock-capturing finite-volume method is employed to discretize the two-fluid flow equations. In here the HLLC approximate Riemann solver is used in evaluating both the convective operator and the source term. To achieve higher-order accuracy we employ the MUSCL approach, which requires a limiter function for positivity. We introduced a novel limiter function and showed that it has better positivity properties than the commonly used ones. It is shown to have higher than second-order convergence. A thirdorder accurate explicit Runge-Kutta method is applied for time integration.

Two types of test cases are considered: a one-dimensional wave-impact problem (the generalized Bagnold model) and a two-dimensional shock-bubble interaction problem (the Haas and Sturtevant experiment).

The generalized Bagnold model is a one-dimensional simplification of a breaking wave. It allows for an analytic solution, provided that the liquid is considered incompressible. For wave impacts where the Bagnold number, describing the severity of the impact, is low, this compared well with the two-fluid model. The ex- 
pected divergence of the two models for higher impact numbers is shown.

An often-used benchmark case for two-dimensional compressible two-fluid flow is provided by experiments conducted by Haas and Sturtevant. The numerical method shows a good qualitative agreement with the shadow photographs from the experiments. The method is able to reproduce free-surface instabilities. The next step is to extend this model to include phase transition using source terms. We think that the improved positivity property of the newly proposed limiter function will prove its worth there, since positivity of the volume fraction is harder to maintain when there are source terms that affect it.

\section{Acknowledgement}

This work is part of the research programme SLING with project number P14-10.1, which is (partly) financed by the Netherlands Organisation for Scientific Research (NWO).

The authors thank the two reviewers of the original version of the manuscript for their constructive comments.

\section{Supplementary material}

Supplementary material associated with this article can be found, in the online version, at doi:10.1016/j.compfluid.2019. 104272.

\section{References}

[1] Baer MR, Nunziato JW. Two-phase mixture theory for the deflagration-to-detonation transition (ddt) in reactive granular materials. Int J Multiphase Flow 1986;12:861-89.

[2] Bagnold RA. Interim report on wave-pressure research. J Inst Civil Eng 1939;12:202-26.

[3] Batten P, Clarke N, Lambert C, Causon D. On the choice of wavespeeds for the HLLC Riemann solver. SIAM J Sci Comput 1997;18:1553-70.

[4] Bogaert H. An experimental investigation of sloshing impact physics in membrane LNG tanks on floating structures. Delft University of Technology; 2018. Ph.D. thesis.

[5] Bogaert H, Léonard S, Brosset L, Kaminski M. Sloshing and scaling: results from the Sloshel project. ISOPE, Beijing; 2010.

[6] Brosset L, Ghidaglia J-M, Guilcher P-M, Le Tarnec L. Generalized Bagnold model. ISOPE, Anchorage; 2013.

[7] Chalons C, Girardin M, Kokh S. An all-regime Lagrange-Projection like scheme for 2D homogeneous models for two-phase flows on unstructured meshes. J Comput Phys 2017;335:885-904.

[8] Crouzet F, Daude F, Galon P, Hérard J-M, Hurisse O, Liu Y. Validation of a two-fluid model on unsteady liquid-vapor water flows. Comput Fluids 2015;119:131-42.
[9] Daude F, Galon P, Gao Z, Blaud E. Numerical experiments using a HLLC-type scheme with ALE formulation for compressible two-phase flows five-equation models with phase transition. Comput Fluids 2014;94:112-38.

[10] de Böck R, Tijsseling A, Koren B. State-of-the-art finite-volume discretisation of Kapila's two-fluid flow model. In: 7th European Conference on Computational Fluid Dynamics (ECCM-ECFD 2018), Glasgow; 2018.

[11] De Lorenzo M, Pelanti M, Lafon Ph. HLLC-Type and path-conservative schemes for a single-velocity six-equation two-phase flow model: a comparative study. Appl Math Comput 2018;333:95-117.

[12] Dias F, Brosset L. Comparative numerical study: description of the calculation cases. ISOPE, Beijing; 2010.

[13] Godunov S. A finite difference method for the computation of discontinuous solutions. Mat Sbornik 1959;47:357-93.

[14] Haas JF, Sturtevant B. Interaction of weak shock waves with cylindrical and spherical gas inhomogeneities. J Fluid Mech 1987;181:41-76.

[15] Hundsdorfer W, Koren B, van Loon M, Verwer JG. A positive finite-difference advection scheme. J Comput Phys 1995:117:35-46.

[16] Johnsen E, Colonius T. Implementation of WENO schemes in compressible multicomponent flow problems. J Comput Phys 2006;219:715-32.

[17] Kapila AK, Menikoff R, Bdzil JB, Son SF, Stewart DS. Two-phase modeling of deflagration-to-detonation transition in granular materials: reduced equations. Phys Fluids 2001;13:3002-24.

[18] Koren B. A robust upwind discretization method for advection, diffusion and source terms. Chapter 5 in notes on numerical fluid mechanics, Vieweg, 45: 1993.

[19] Kreeft JJ, Koren B. A new formulation of Kapila's five-equation model for compressible two-fluid flow, and its numerical treatment. J Comput Phys 2010;229:6220-42.

[20] LeVeque R. Finite-volume methods for hyperbolic problems. Cambridge texts in applied mathematics. Cambridge University Press; 2002.

[21] Quirk JJ, Karni S. On the dynamics of shock-bubble interaction. J Fluid Mech 1996;318:129-63.

[22] Ramkema C. A model law for wave impacts on coastal structures. In: 16th Conference on coastal engineering, Hamburg; 1978

[23] Rodio MG, Abgrall R. An innovative phase transition modeling for reproducing cavitation through a five-equation model and theoretical generalization to six and seven-equation models. Int J Heat Mass Transf 2015;89:1386-401.

[24] Saurel R, Boivin P, Le Métayer O. A general formulation for cavitating, boiling and evaporating flows. Comput Fluid 2016;128:53-64.

[25] Shu C, Osher S. Efficient implementation of essentially non-oscillatory shockcapturing schemes. J Comput Phys 1988;77:439-71.

[26] Spekreijse S. Multigrid solution of monotone second-order discretizations of hyperbolic conservation laws. Math Comput 1987;49:135-55.

[27] Sweby PK. High resolution schemes using flux limiters for hyperbolic conservation laws. SIAM J Numer Anal 1984:21:995-1011.

[28] Tijsseling AS, Hou Q, Bozkuş Z. Analytical solutions for liquid slugs and pigs traveling in pipelines with entrapped gas. In: ASME pressure vessels and piping conference, Waikoloa, Hawaii, PVP Vol 4; 2017.

[29] Toro EF. Riemann solvers and numerical methods for fluid dynamics. Springer; 2009.

[30] van Leer B. Towards the ultimate conservative difference scheme V. A second-order sequel to Godunov's method. J Comput Phys 1979;32:101-36.

[31] Wackers J, Koren B. Five-equation model for compressible two-fluid flow. Report MAS-E0414. CWI; 2004.

[32] Wood AB. A textbook of sound. G. Bell and Sons; 1930. 